\title{
Periodic Oscillatory Phenomenon in Fractional-Order Neural Networks Involving Different Types of Delays
}

\author{
Nengfa Wang, ${ }^{1}$ Changjin $X u \mathbb{D}^{2}$ and Zixin Liu ${ }^{1}$ \\ ${ }^{1}$ School of Mathematics and Statistics, Guizhou University of Finance and Economics, Guiyang 550025, China \\ ${ }^{2}$ Guizhou Key Laboratory of Economics System Simulation, Guizhou University of Finance and Economics, \\ Guiyang 550025, China \\ Correspondence should be addressed to Changjin Xu; xcj403@126.com
}

Received 25 May 2021; Revised 29 July 2021; Accepted 4 October 2021; Published 25 October 2021

Academic Editor: Fahd Jarad

Copyright (c) 2021 Nengfa Wang et al. This is an open access article distributed under the Creative Commons Attribution License, which permits unrestricted use, distribution, and reproduction in any medium, provided the original work is properly cited.

\begin{abstract}
This research is chiefly concerned with the stability and Hopf bifurcation for newly established fractional-order neural networks involving different types of delays. By means of an appropriate variable substitution, equivalent fractional-order neural network systems involving one delay are built. By discussing the distribution of roots of the characteristic equation of the established fractional-order neural network systems and selecting the delay as bifurcation parameter, a novel delay-independent bifurcation condition is derived. The investigation verifies that the delay is a significant parameter which has an important influence on stability nature and Hopf bifurcation behavior of neural network systems. The computer simulation plots and bifurcation graphs effectively illustrate the reasonableness of the theoretical fruits.
\end{abstract}

\section{Introduction}

It is widely known that neural network systems own great application value in plenty of areas such as disease treatment, pattern recognition, intelligent control, biology, information processing, control technique, and so on [1-6]. In real world, time delay usually occurs in lots of neural networks and biological systems since there is delay of information transmission of distinct neurons and the response of diverse biotic populations. Therefore, grasping the effect of delay on the dynamic law for many dynamical models is a key topic in contemporary science. Generally speaking, the delay frequently leads to the disappearance of stability and the appearance of bifurcation, chaotic behavior, and a lot of other dynamic characteristics. So, a number of researchers pay much attention to various neural networks, and lots of excellent results have been reported. For instance, Lin and Zhang [7] considered the global asymptotic synchronization of delayed BAM neural networks by means of the LMI method and matrix measure method. Ding et al. [8] established a sufficient criterion guaranteeing the global robust exponential stability of BAM neural networks involving discrete delay and the distributed delay under uncertainty by applying an appropriate Lyapunov-Krasovskii function. In the work of Hou et al. [9], the authors dealt with the stability issue of discrete-time uncertain models concerning Markov jump and delay. In 2021, Zhou [10] investigated the global exponential dissipativity for recurrent neural networks involving proportional delays. For more works on this subject, one can see [11-13].

It should be noted that all the involved publications mentioned above only focus on integer-order neural networks concerning delays and are not concerned with the fractional-order form. The research on fractional-order differential equation has remained a relatively slow development situation because of the lack of theoretical body of knowledge and realistic background. Until recently, fractional-order differential dynamical system has aroused widespread interest from many scholars since it owns great application prospect in a lot of fields such as control technique, various physical waves, neural network models, biological systems, and so on $[14,15]$. Many scholars argue that that fractional-order differential model can more efficaciously characterize the actual situation in object world 
than the traditional integer-order differential model since it has preferable memory trait and hereditary property [16]. Recently, a lot of scholars have dedicated themselves to the study on dynamical behavior for fractional-order differential models. In particular, the research on fractional-order neural networks concerning delays has aroused widespread interest of many researchers and a lot of results on fractional-order neural networks concerning delays have been published (see [17-19]).

Delay-induced bifurcation is an important topic in neural network area. Over the past few decades, a number of works on delay-induced bifurcation of integer-order neural networks have been reported and the basic bifurcation theory is sound. It is a pity that the study on Hopf bifurcation for fractional-order neural networks is relatively rare. In recent years, some researchers pay much attention to Hopf bifurcation of fractional-order delayed neural networks and some good results have been achieved. For instance, Xu et al. [20] made a detailed analysis on the stability and the onset of Hopf bifurcation for fractional-order BAM neural networks involving time delays. Djilali et al. [21] discussed the
Turing-Hopf bifurcation for a fractional-order diffusive mussel-algae model. $\mathrm{Hu}$ et al. [22] investigated the Hopf bifurcation and chaos for a fractional-order memristorbased chaotic circuit system involving delays. Xu et al. [23] dealt with Hopf bifurcation of fractional-order BAM neural network models involving multiple delays. For more studies on this aspect, one can see $[24,25]$.

It is worth noticing that in many cases, the study on bifurcation of fractional-order delayed neural networks is only concerned with discrete delay. Considering that in neural network systems, there are numerous parallel paths involving many diverse axon sizes and lengths [26], it is very important for us to introduce continuous distributed delays into neural networks to depict the signal transmission of neurons. In order to grasp the impact of the distributed delay on Hopf bifurcation for neural networks concerning time delay, plenty of authors have dedicated themselves to all kinds of neural networks involving distributed delays (see $[27,28])$. In 2016, Karaoğlu et al. [29] analyzed the following neural networks concerning mixed delays:

$$
\left\{\begin{array}{l}
\frac{\mathrm{d} v_{1}(t)}{\mathrm{d} t}=-v_{1}(t)+\gamma_{11} h_{11}\left(\int_{-\infty}^{t} H(t-s) v_{1}(s) \mathrm{d} s\right)+\gamma_{12} h_{12}\left(v_{2}\left(t-\eta_{2}\right)\right) \\
\frac{\mathrm{d} v_{2}(t)}{\mathrm{d} t}=-v_{2}(t)+\gamma_{21} h_{21}\left(v_{1}\left(t-\eta_{1}\right)\right)+\gamma_{22} h_{22}\left(\int_{-\infty}^{t} H(t-s) v_{2}(s) \mathrm{d} s\right)
\end{array}\right.
$$

where $v_{l}(t), \quad(l=1,2)$, stands for the state of the $l$ th neuron at time $t, \gamma_{i j}, \quad(i, j=1,2)$, denotes the connection weight and is real constant, $\eta_{l}>0, \quad(l=1,2)$, denotes the delay, and $H($.$) denotes nonnegative bounded delay kernel$ defined on $[0, \infty)$, which reflects the impact of the past states on the present dynamics [29]. Usually, the kernel function takes the following two forms:

(1) $\int_{-\infty}^{t} H(\mu) \mathrm{d} \mu=1, H(\mu)=\beta e^{-\beta(t-\mu)}, \quad \beta>0$.

(2) $\int_{-\infty}^{t} H(t-\mu) \mathrm{d} \mu=1, H(\mu)=\beta e^{-\beta \mu}, \quad \beta>0$.

In [29], Karaoğlu et al. took kernel function as case (2). By means of stability criterion and Hopf bifurcation theory of delayed differential equation, Karaoğlu et al. obtained a sufficient condition to guarantee the stability and the onset of Hopf bifurcation of system (1). In addition, with the aid of center manifold theorem and normal form theory, the concrete formula to determine bifurcation nature is given.

Motivated by the discussion above, our concern is the stability and Hopf bifurcation for fractional-order neural networks involving discrete delays and distributed delays. On the basis of the work of Karaoğlu et al. [29], in this work, we modify system (1) as the following fractional-order version:

$$
\left\{\begin{array}{l}
\frac{\mathrm{d} v_{1}^{\rho}(t)}{\mathrm{d} t^{\rho}}=-v_{1}(t)+\gamma_{11} h_{11}\left(\int_{-\infty}^{t} H(t-s) v_{1}(s) \mathrm{d} s\right)+\gamma_{12} h_{12}\left(v_{2}\left(t-\eta_{2}\right)\right), \\
\frac{\mathrm{d} v_{2}^{\rho}(t)}{\mathrm{d} t^{\rho}}=-v_{2}(t)+\gamma_{21} h_{21}\left(v_{1}\left(t-\eta_{1}\right)\right)+\gamma_{22} h_{22}\left(\int_{-\infty}^{t} H(t-s) v_{2}(s) \mathrm{d} s\right),
\end{array}\right.
$$

where $0<\rho<1$ is a constant, $v_{l}(t), \quad(l=1,2)$, stands for the state of the $l$ th neuron at time $t, \gamma_{i j}, \quad(i, j=1,2)$, denotes the connection weight and is real constant, $\eta_{l}>0, \quad(l=1,2)$, denotes the delay, and $H($.$) denotes$ nonnegative bounded delay kernel defined on $[0, \infty)$, which reflects the impact of the past states on the present dynamics [29], and the kernel function $H($.$) takes$ form (2). 
The novelty of this paper lies in constructing novel fractional-order neural networks including fractional-order and integer-order equations by applying an appropriate variable substitution. Up to now, there are very few publications on this topic.

In order to establish the prime results of this work, the following assumptions are needed:

$$
\begin{aligned}
& \left(A_{1}\right) h_{k l} \in C, h_{k l}(0)=0, \quad(k, l=1,2) . \\
& \left(A_{2}\right) \eta_{1}+\eta_{2}=\eta .
\end{aligned}
$$

This paper is organized as follows. Section 2 gives some essential theories on fractional-order differential equation. Section 3 gives the bifurcation results for system (2) with kernel function (2). Section 4 carries out computer simulation to sustain the reasonableness of the derived main results. This research ends in Section 5.

\section{Basic Theory on Fractional-Order Differential Equation}

In this segment, we will make some preparations on some necessary theories on fractional-order differential equation.
Definition 1 (see [30]). Define Caputo-type fractional-order derivative as follows:

$$
\mathscr{D}^{\rho} w(\zeta)=\frac{1}{\Gamma(\kappa-\rho)} \int_{\zeta_{0}}^{\zeta} \frac{w^{(\kappa)}(\nu)}{(\zeta-v)^{\rho-\kappa+1}} \mathrm{~d} v,
$$

where $w(\zeta) \in\left(\left[\zeta_{0}, \infty\right), R\right), \Gamma(v)=\int_{0}^{\infty} \zeta^{v-1} e^{-\zeta} \mathrm{d} \zeta, \quad \zeta \geq \zeta_{0}$, $\kappa \in Z^{+}, \kappa-1 \leq \rho<\kappa$.

Lemma 1 (see [31, 32]). Consider the following system:

$$
\frac{\mathrm{d}^{\rho} u(t)}{\mathrm{d} t^{\rho}}=g(t, u(t)), u(0)=u_{0},
$$

where $\rho \in(0,1]$ and $g(t, u(t)): R^{+} \times R^{n} \longrightarrow R^{n}$. Denote $u_{0}$ as the equilibrium point of (4). Then, $u_{0}$ is locally asymptotically stable if every eigenvalue $\mu$ of $\left.(\partial g(t, u) / \partial u)\right|_{u=u_{0}}$ satisfies $|\arg (\mu)|>\rho \pi / 2$.

Lemma 2 (see [33]). Consider the following system:

$$
\left\{\begin{array}{l}
\frac{\mathrm{d}^{\rho_{1}} \mathscr{B}_{1}(t)}{\mathrm{d} t^{\rho_{1}}}=q_{11} \mathscr{B}_{1}\left(t-\eta_{11}\right)+q_{12} \mathscr{B}_{2}\left(t-\eta_{12}\right)+, \cdots,+q_{1 k} \mathscr{B}_{k}\left(t-\eta_{1 k}\right), \\
\frac{\mathrm{d}^{\rho_{2}} \mathscr{B}_{2}(t)}{\mathrm{d} t^{\rho_{2}}}=q_{21} \mathscr{B}_{1}\left(t-\eta_{21}\right)+q_{22} \mathscr{B}_{2}\left(t-\eta_{22}\right)+, \cdots,+q_{2 k} \mathscr{B}_{k}\left(t-\eta_{2 k}\right), \\
\vdots \\
\frac{\mathrm{d}^{\rho_{k}} \mathscr{B}_{k}(t)}{\mathrm{d} t^{\rho_{k}}}=q_{k 1} \mathscr{B}_{1}\left(t-\eta_{k 1}\right)+q_{k 2} \mathscr{B}_{2}\left(t-\eta_{k 2}\right)+, \cdots,+q_{k k} \mathscr{B}_{k}\left(t-\eta_{k k}\right),
\end{array}\right.
$$

where $\rho_{h} \in(0,1), \quad(h=1,2, \ldots, k)$. Set

$$
\Delta(s)=\left[\begin{array}{cccc}
s^{\rho_{1}}-q_{11} e^{-s \eta_{11}} & -q_{12} e^{-s \eta_{12}} & \cdots & -q_{1 k} e^{-s \eta_{1 k}} \\
-q_{21} e^{-s \eta_{12}} & s^{\rho_{2}}-q_{22} e^{-s \eta_{22}} & \cdots & -q_{2 k} e^{-s \eta_{2 k}} \\
\vdots & \vdots & \ddots & \vdots \\
-p_{k 1} e^{-s \eta_{k 1}} & -q_{k 2} e^{-s \eta_{k 2}} & \cdots & s^{\rho_{k}}-q_{k k} e^{-s \eta_{k k}}
\end{array}\right] .
$$

The zero solution of system (5) is said to be asymptotically stable if every root of $\operatorname{det}(\Delta(s))=0$ owns negative real parts.

Give the system:

$$
\left\{\begin{array}{l}
\frac{\mathrm{d}^{\rho_{1}} \mathscr{B}_{1}(t)}{\mathrm{d} t^{\rho_{1}}}=q_{11} \mathscr{B}_{1}(t)+q_{12} \mathscr{B}_{2}(t)+, \cdots,+q_{1 k} \mathscr{B}_{k}(t), \\
\frac{\mathrm{d}^{\rho_{2}} \mathscr{B}_{2}(t)}{\mathrm{d} t^{\rho_{2}}}=q_{21} \mathscr{B}_{1}(t)+q_{22} \mathscr{B}_{2}(t)+, \cdots,+q_{2 k} \mathscr{B}_{k}(t), \\
\vdots \\
\frac{\mathrm{d}^{\rho_{k}} \mathscr{B}_{k}(t)}{\mathrm{d} t^{\rho_{k}}}=q_{k 1} \mathscr{B}_{1}(t)+q_{k 2} \mathscr{B}_{2}(t)+, \cdots,+q_{k k} \mathscr{B}_{k}(t),
\end{array}\right.
$$


where $\varrho_{h} \in(0,1], \quad(h=1,2, \ldots, k)$. The characteristic equation of system (7) takes the following form:

$$
\operatorname{det}\left[\begin{array}{cccc}
s^{\rho_{1}}-q_{11} & -q_{12} & \cdots & -q_{1 k} \\
-q_{21} & s^{\rho_{2}}-q_{22} & \cdots & -q_{2 k} \\
\vdots & \vdots & \ddots & \vdots \\
-q_{k 1} & -q_{k 2} & \cdots & s^{\rho_{k}}-q_{k k}
\end{array}\right]=0 .
$$

Suppose that $\psi_{j}=\alpha_{j} / \beta_{j}, \alpha_{j}, \beta_{j} \in Z^{+},\left(\alpha_{j}, \beta_{j}\right)=1$ and $\beta$ is the lowest common multiple of $\beta_{j}$ of $\psi_{j}, \quad j=1,2, \ldots, k$.

Lemma 3 (see [33]). We say that the zero solution of system (7) is locally asymptotically stable if every root $\lambda$ s of the following equation:

$$
\operatorname{det}\left[\begin{array}{cccc}
\lambda^{\beta \psi_{1}}-q_{11} & -q_{12} e^{-s \eta_{12}} & \cdots & -q_{1 k} \\
-q_{21} & \lambda^{\beta \psi_{2}}-q_{22} & \cdots & -q_{2 k} \\
\vdots & \vdots & \ddots & \vdots \\
-q_{k 1} & -q_{k 2} & \cdots & \lambda^{\beta \psi_{k}}-q_{k k}
\end{array}\right]=0,
$$

obeys $|\arg (\lambda)|>(\pi / 2 \beta)$.

\section{Bifurcation Study for Neural Networks (2)}

In this segment, we will discuss the stability behavior and the emergence of Hopf bifurcation for neural networks (2). Let

$$
\left\{\begin{array}{l}
v_{3}(t)=\int_{-\infty}^{t} H(t-s) v_{1}(s) \mathrm{d} s=\int_{-\infty}^{t} \beta e^{\beta(t-s)} v_{1}(s) \mathrm{d} s, \\
v_{4}(t)=\int_{-\infty}^{t} H(t-s) v_{2}(s) \mathrm{d} s=\int_{-\infty}^{t} \beta e^{\beta(t-s)} v_{2}(s) \mathrm{d} s,
\end{array}\right.
$$

and then

$$
\left\{\begin{array}{l}
\frac{\mathrm{d} v_{3}(t)}{\mathrm{d} t}=\left[\int_{-\infty}^{t} \beta e^{\beta(t-s)} v_{1}(s) \mathrm{d} s\right]^{\prime}=-\beta v_{3}(t)+\beta v_{1}(t), \\
\frac{\mathrm{d} v_{4}(t)}{\mathrm{d} t}=\left[\int_{-\infty}^{t} \beta e^{\beta(t-s)} v_{2}(s) \mathrm{d} s\right]^{\prime}=-\beta v_{4}(t)+\beta v_{2}(t) .
\end{array}\right.
$$

Thus, system (2) becomes the following equivalent form:

$$
\left\{\begin{array}{l}
\frac{\mathrm{d} \nu_{1}^{\rho}(t)}{\mathrm{d} t^{\rho}}=-v_{1}(t)+\gamma_{11} h_{11}\left(v_{3}(t)\right)+\gamma_{12} h_{12}\left(v_{2}\left(t-\eta_{2}\right)\right), \\
\frac{\mathrm{d} \nu_{2}^{\rho}(t)}{\mathrm{d} t^{\rho}}=-v_{2}(t)+\gamma_{21} h_{21}\left(v_{1}\left(t-\eta_{1}\right)\right)+\gamma_{22} h_{22}\left(v_{4}(t)\right), \\
\frac{\mathrm{d} v_{3}(t)}{\mathrm{d} t}=-\beta v_{3}(t)+\beta v_{1}(t), \\
\frac{\mathrm{d} v_{4}(t)}{\mathrm{d} t}=-\beta v_{4}(t)+\beta v_{2}(t) .
\end{array}\right.
$$

By virtue of $\left(A_{1}\right)$, one easily knows that system (12) has a unique zero equilibrium point. The linear system of equation (12) near the zero equilibrium point takes the following expression:

$$
\left\{\begin{array}{l}
\frac{\mathrm{d} v_{1}^{\rho}(t)}{\mathrm{d} t^{\rho}}=-v_{1}(t)+\varrho_{11} v_{3}(t)+\varrho_{12} v_{2}\left(t-\eta_{2}\right) \\
\frac{\mathrm{d} v_{2}^{\rho}(t)}{\mathrm{d} t^{\rho}}=-v_{2}(t)+\varrho_{21} v_{1}\left(t-\eta_{1}\right)+\varrho_{22} v_{4}(t) \\
\frac{\mathrm{d} v_{3}(t)}{\mathrm{d} t}=-\beta v_{3}(t)+\beta v_{1}(t) \\
\frac{\mathrm{d} v_{4}(t)}{\mathrm{d} t}=-\beta v_{4}(t)+\beta v_{2}(t)
\end{array}\right.
$$

where $\varrho_{k l}=\gamma_{k l} h_{k l}^{\prime}(0), \quad(k, l=1,2) . \quad$ The characteristic equation for (13) is given by

$$
\operatorname{det}\left[\begin{array}{cccc}
s^{\rho}+1 & -\varrho_{12} e^{-s \eta_{2}} & -\varrho_{11} & 0 \\
-\varrho_{21} e^{-s \eta_{1}} & s^{\rho}+1 & 0 & -\varrho_{22} \\
-\beta & 0 & s+\beta & 0 \\
0 & -\beta & 0 & s+\beta
\end{array}\right]=0 .
$$

Let $\rho=(\zeta / \xi)$, where $\zeta, \xi \in Z^{+}$and $(\zeta, \xi)=1$. Denote $\lambda=s^{(1 / \xi)}$. If $\eta=0$, which implies that $\eta_{1}=\eta_{2}=0$. Then, equation (14) takes the following form:

$$
\operatorname{det}\left[\begin{array}{cccc}
s^{\rho}+1 & -\varrho_{12} & -\varrho_{11} & 0 \\
-\varrho_{21} & s^{\rho}+1 & 0 & -\varrho_{22} \\
-\beta & 0 & s+\beta & 0 \\
0 & -\beta & 0 & s+\beta
\end{array}\right]=0
$$


Lemma 4. For system (12), if $\eta=0$ and each root $\lambda$ of equation (15) satisfies $|\arg (\lambda)|>(\pi / 2 \xi)$, then the zero equilibrium point of system (12) is locally asymptotically stable in Lyapunov sense.

Proof. It is easy to see that if $\eta=0$, then the characteristic equation (14) can be changed into equation (15). Applying Lemma 3, we can conclude that Lemma 4 is true.

By equation (14), we obtain

$$
\begin{aligned}
s^{2 \rho+2} & +\epsilon_{1} s^{2 \rho+1}+\epsilon_{2} s^{2 \rho}+2 s^{\rho+2}+\epsilon_{3} s^{\rho+1}+\epsilon_{4} s^{\rho}+s^{2}+\epsilon_{5} s+\epsilon_{6} \\
& +\left(\epsilon_{7} s^{2}+\epsilon_{8} s+\epsilon_{9}\right) e^{-2 s \eta}=0,
\end{aligned}
$$

where

$$
\left\{\begin{array}{l}
\epsilon_{1}=2 \beta, \\
\epsilon_{2}=\beta, \\
\epsilon_{3}=\beta\left(\varrho_{11}-\varrho_{22}+4\right), \\
\epsilon_{4}=2 \beta+\beta^{2} \varrho_{11}-\beta^{2} \varrho_{22}, \\
\epsilon_{5}=\beta\left(\varrho_{11}-\varrho_{22}+2\right), \\
\epsilon_{6}=\beta+\beta^{2}\left(\varrho_{11}-\varrho_{22}\right), \\
\epsilon_{7}=-\varrho_{12} \varrho_{21}, \\
\epsilon_{8}=-2 \beta \varrho_{12} \varrho_{21}, \\
\epsilon_{9}=-\beta^{2} \varrho_{12} \varrho_{21} .
\end{array}\right.
$$

Suppose that $s=i \sigma=\sigma(\cos (\pi / 2)+i \sin (\pi / 2))$ is the root of equation (16); then, we have

$$
\begin{aligned}
& {\left[\sigma^{2 \rho+2}\left(\cos \frac{(2 \rho+2) \pi}{2}+i \sin \frac{(2 \rho+2) \pi}{2}\right)+\epsilon_{1} \sigma^{2 \rho+1}\left(\cos \frac{(2 \rho+1) \pi}{2}+i \sin \frac{(2 \rho+1) \pi}{2}\right)\right.} \\
& +\epsilon_{2} \sigma^{2 \rho}(\cos \rho \pi+i \sin \rho \pi)+2 \sigma^{\rho+2}\left(\cos \frac{(\rho+2) \pi}{2}+i \sin \frac{(\rho+2) \pi}{2}\right) \\
& \left.+\epsilon_{3} \sigma^{\rho+1}\left(\cos \frac{(\rho+1) \pi}{2}+i \sin \frac{(\rho+1) \pi}{2}\right)+\epsilon_{4} \sigma^{\rho}\left(\cos \frac{\rho \pi}{2}+i \sin \frac{\rho \pi}{2}\right)-\sigma^{2}+i \epsilon_{5} \sigma+\epsilon_{6}\right] \\
& +\left(-\epsilon_{7} \sigma^{2}+i \epsilon_{8} \sigma+\epsilon_{9}\right)(\cos 2 \sigma \eta-i \sin 2 \sigma \eta)=0 .
\end{aligned}
$$

By virtue of equation (18), one gets

where

$$
\left\{\begin{array}{l}
Q_{1} \cos 2 \sigma \eta+Q_{2} \sin 2 \sigma \eta=Q_{3}, \\
Q_{2} \cos 2 \sigma \eta-Q_{1} \sin 2 \sigma \eta=Q_{4},
\end{array}\right.
$$

$$
\left\{\begin{array}{l}
Q_{1}=\epsilon_{9}-\epsilon_{7} \sigma^{2}, \\
Q_{2}=\epsilon_{8} \sigma \\
Q_{3}=-\sigma^{2 \rho+2} \cos \frac{(2 \rho+2) \pi}{2}-\epsilon_{1} \sigma^{2 \rho+1} \cos \frac{(2 \rho+1) \pi}{2}-\epsilon_{2} \sigma^{2 \rho} \cos \rho \pi-2 \sigma^{\rho+2} \cos \frac{(\rho+2) \pi}{2}-\epsilon_{3} \sigma^{\rho+1} \cos \frac{(\rho+1) \pi}{2}-\epsilon_{4} \sigma^{\rho} \cos \frac{\rho \pi}{2}+\sigma^{2}-\epsilon_{6}, \\
Q_{4}=-\sigma^{2 \rho+2} \sin \frac{(2 \rho+2) \pi}{2}-\epsilon_{1} \sigma^{2 \rho+1} \sin \frac{(2 \rho+1) \pi}{2}-\epsilon_{2} \sigma^{2 \rho} \sin \rho \pi-2 \sigma^{\rho+2} \sin \frac{(\rho+2) \pi}{2}-\epsilon_{3} \sigma^{\rho+1} \sin \frac{(\rho+1) \pi}{2}-\epsilon_{4} \sigma^{\rho} \sin \frac{\rho \pi}{2}-\epsilon_{5} \sigma .
\end{array}\right.
$$

By (19), we have

$$
\cos 2 \sigma \eta=\frac{\mathbb{Q}_{1} Q_{3}+Q_{2} Q_{4}}{Q_{1}^{2}+Q_{2}^{2}}
$$

and

$$
Q_{1}^{2}+Q_{2}^{2}=Q_{3}^{2}+Q_{4}^{2} .
$$

In equation (20), denote 


$$
\left\{\begin{array}{l}
\alpha_{1}=-\cos \frac{(2 \rho+2) \pi}{2} \\
\alpha_{2}=-\epsilon_{1} \cos \frac{(2 \rho+1) \pi}{2} \\
\alpha_{3}=-\epsilon_{2} \cos \rho \pi \\
\alpha_{4}=-2 \cos \frac{(\rho+2) \pi}{2} \\
\alpha_{5}=-\epsilon_{3} \cos \frac{(\rho+1) \pi}{2} \\
\alpha_{6}=-\epsilon_{4} \cos \frac{\rho \pi}{2}, \\
\alpha_{12}=-\epsilon_{4} \sin \frac{\rho \pi}{2} \\
\alpha_{10}=-2 \sin \frac{(2 \rho+2) \pi}{2} \\
\alpha_{9}=-\epsilon_{2} \sin \frac{\rho \pi}{2} \sin \frac{(2 \rho+1) \pi}{2}
\end{array}\right.
$$

and then (20) becomes

$$
\left\{\begin{array}{l}
\mathbb{Q}_{1}=\epsilon_{9}-\epsilon_{7} \sigma^{2}, \\
\mathbb{Q}_{2}=\epsilon_{8} \sigma, \\
\mathcal{Q}_{3}=\alpha_{1} \sigma^{2 \rho+2}+\alpha_{2} \sigma^{2 \rho+1}+\alpha_{3} \sigma^{2 \rho}+\alpha_{4} \sigma^{\rho+2}+\alpha_{5} \sigma^{\rho+1}+\alpha_{6} \sigma^{\rho}+\sigma^{2}-\epsilon_{6}, \\
\mathbb{Q}_{4}=\alpha_{7} \sigma^{2 \rho+2}+\alpha_{8} \sigma^{2 \rho+1}+\alpha_{9} \sigma^{2 \rho}+\alpha_{10} \sigma^{\rho+2}+\alpha_{11} \sigma^{\rho+1}+\alpha_{12} \sigma^{\rho}-\epsilon_{5} \sigma .
\end{array}\right.
$$

According to (22) and (24), we get

$$
\begin{aligned}
\varsigma_{1} \sigma^{4 \rho+4} & +\varsigma_{2} \sigma^{4 \rho+3}+\varsigma_{3} \sigma^{4 \rho+2}+\varsigma_{4} \sigma^{4 \rho+1}+\varsigma_{5} \sigma^{4 \rho}+\varsigma_{6} \sigma^{3 \rho+4}+\varsigma_{7} \sigma^{3 \rho+3}+\varsigma_{8} \sigma^{3 \rho+2}+\varsigma_{9} \sigma^{3 \rho+1}+\varsigma_{10} \sigma^{3 \rho}+\varsigma_{11} \sigma^{2 \rho+4}+\varsigma_{12} \sigma^{2 \rho+3} \\
& +\varsigma_{13} \sigma^{2 \rho+2}+\varsigma_{14} \sigma^{2 \rho+1}+\varsigma_{15} \sigma^{2 \rho}+\varsigma_{16} \sigma^{\rho+4}+\varsigma_{17} \sigma^{\rho+3}+\varsigma_{18} \sigma^{\rho+2}+\varsigma_{19} \sigma^{\rho+1}+\varsigma_{20} \sigma^{\rho}+\varsigma_{21} \sigma^{4}+\varsigma_{22} \sigma^{2}+\varsigma_{23}=0,
\end{aligned}
$$

where 


$$
\left\{\begin{array}{l}
\varsigma_{1}=\alpha_{1}^{2}+\alpha_{7}^{2} \\
\varsigma_{2}=2\left(\alpha_{1} \alpha_{2}+\alpha_{7} \alpha_{8}\right) \\
\varsigma_{3}=\alpha_{2}^{2}+\alpha_{8}^{2}+2\left(\alpha_{1} \alpha_{3}+\alpha_{7} \alpha_{9}\right) \\
\varsigma_{4}=2\left(\alpha_{2} \alpha_{3}+\alpha_{8} \alpha_{9}\right) \\
\varsigma_{5}=\alpha_{3}^{2}+\alpha_{9}^{2} \\
\varsigma_{6}=2\left(\alpha_{1} \alpha_{4}+\alpha_{7} \alpha_{10}\right) \\
\varsigma_{7}=2\left(\alpha_{1} \alpha_{5}+\alpha_{2} \alpha_{4}+\alpha_{7} \alpha_{11}+\alpha_{8} \alpha_{10}\right) \\
\varsigma_{8}=2\left(\alpha_{1} \alpha_{6}+\alpha_{2} \alpha_{5}+\alpha_{3} \alpha_{4}+\alpha_{7} \alpha_{12}+\alpha_{8} \alpha_{11}+\alpha_{9} \alpha_{10}\right) \\
\varsigma_{9}=2\left(\alpha_{2} \alpha_{6}+\alpha_{3} \alpha_{5}+\alpha_{8} \alpha_{12}+\alpha_{9} \alpha_{11}\right) \\
\varsigma_{10}=2\left(\alpha_{3} \alpha_{6}+\alpha_{9} \alpha_{12}\right) \\
\varsigma_{11}=\alpha_{4}^{2}+\alpha_{10}^{2}+2 \alpha_{1} \\
\varsigma_{12}=2\left(\alpha_{2}+\alpha_{4} \alpha_{5}+\alpha_{10} \alpha_{11}\right)-\alpha_{7} \epsilon_{5} \\
\varsigma_{13}=\alpha_{5}^{2}+\alpha_{11}^{2}+2\left(\alpha_{3}-\alpha_{1} \alpha_{6}-\alpha_{8} \epsilon_{5}+\alpha_{10} \alpha_{12}+\alpha_{4} \alpha_{6}\right) \\
\varsigma_{14}=2\left(\alpha_{5} \alpha_{6}+\alpha_{2} \epsilon_{6}+\alpha_{11} \alpha_{12}-\alpha_{9} \epsilon_{5}\right) \\
\varsigma_{15}=\alpha_{6}^{2}-2 \alpha_{3} \epsilon_{6}+\alpha_{12} \\
\varsigma_{16}=2 \alpha_{4}, \\
\varsigma_{17}=2\left(\alpha_{5}-\alpha_{10} \epsilon_{5}\right) \\
\varsigma_{18}=2\left(\alpha_{6}-\alpha_{4} \epsilon_{6}-\alpha_{11} \epsilon_{5}\right) \\
\varsigma_{19}=-2\left(\alpha_{5} \epsilon_{6}+\alpha_{12} \epsilon_{5}\right) \\
\varsigma_{20}=-2 \alpha_{6} \epsilon_{6}, \\
\varsigma_{21}=1-\epsilon_{72}^{2} \\
\varsigma_{23}=-2 \epsilon_{7}+\epsilon_{5}^{2}-\epsilon_{9}^{2}+2 \epsilon_{7} \epsilon_{9} \\
\end{array}\right.
$$

$$
\begin{aligned}
\mathcal{S}(\sigma)= & \varsigma_{1} \sigma^{4 \rho+4}+\varsigma_{2} \sigma^{4 \rho+3}+\varsigma_{3} \sigma^{4 \rho+2}+\varsigma_{4} \sigma^{4 \rho+1}+\varsigma_{5} \sigma^{4 \rho}+\varsigma_{6} \sigma^{3 \rho+4}+\varsigma_{7} \sigma^{3 \rho+3}+\varsigma_{8} \sigma^{3 \rho+2}+\varsigma_{9} \sigma^{3 \rho+1}+\varsigma_{10} \sigma^{3 \rho}+\varsigma_{11} \sigma^{2 \rho+4}+\varsigma_{12} \sigma^{2 \rho+3} \\
& +\varsigma_{13} \sigma^{2 \rho+2}+\varsigma_{14} \sigma^{2 \rho+1}+\varsigma_{15} \sigma^{2 \rho}+\varsigma_{16} \sigma^{\rho+4}+\varsigma_{17} \sigma^{\rho+3}+\varsigma_{18} \sigma^{\rho+2}+\varsigma_{19} \sigma^{\rho+1}+\varsigma_{20} \sigma^{\rho}+\varsigma_{21} \sigma^{4}+\varsigma_{22} \sigma^{2}+\varsigma_{23} .
\end{aligned}
$$

Now the following assumption is given.

$\left(A_{3}\right) \varsigma_{23}<0$, where $\varsigma_{23}$ is defined by $(26)$.

Lemma 5. If $\left(A_{3}\right)$ holds, then equation (16) possesses at least a pair of purely imaginary roots.

Proof. Clearly, $\mathcal{S}(0)=\varsigma_{23}<0$ and $\lim _{\sigma \longrightarrow \infty} \mathcal{S}(\sigma)=+\infty$; then, equation (25) possesses at least one positive root. That is to say, equation (16) possesses at least a couple of purely imaginary roots.

We cannot easily derive the solution of equation (25) since the powers of equation (25) are fractional number. Thus, we will change the powers of equation (25) to integer powers. Let $y=\sigma^{(1 / \xi)}$; then, $\sigma=y^{\xi}$. In view of equation (25), one has

$$
\begin{array}{r}
\varsigma_{1} y^{4 \zeta+4 \xi}+\varsigma_{2} y^{4 \zeta+3 \xi}+\varsigma_{3} y^{4 \zeta+2 \xi}+\varsigma_{4} y^{4 \zeta+\xi}+\varsigma_{5} y^{4 \zeta}+\varsigma_{6} y^{3 \zeta+4 \xi}+\varsigma_{7} y^{3 \zeta+3 \xi}+\varsigma_{8} y^{3 \zeta+2 \xi}+\varsigma_{9} y^{3 \zeta+\xi}+\varsigma_{10} y^{3 \zeta}+\varsigma_{11} y^{2 \zeta+4 \xi}+\varsigma_{12} y^{2 \zeta+3 \xi} \\
+\varsigma_{13} y^{2 \zeta+2 \xi}+\varsigma_{14} y^{2 \zeta+\xi}+\varsigma_{15} y^{2 \zeta}+\varsigma_{16} y^{\zeta+4 \xi}+\varsigma_{17} y^{\zeta+3 \xi}+\varsigma_{18} y^{\zeta+2 \xi}+\varsigma_{19} y^{\zeta+\xi}+\varsigma_{20} y^{\zeta}+\varsigma_{21} y^{4 \xi}+\varsigma_{22} y^{2 \xi}+\varsigma_{23}=0
\end{array}
$$


With the aid of computer, we can derive the roots of equation (28). Assume that equation (28) possesses the positive root (say $y_{j}$ ); then, equation (25) possesses the positive root $\sigma_{j}=y_{j}^{\xi}$. Assume that equation (28) owns $k$ positive roots $y_{j}, \quad j=1,2, \ldots, k$. By $(21)$, one gets

$$
\begin{aligned}
\eta_{j}^{n} & =\frac{1}{2 \sigma_{j}}\left[\arccos \frac{\mathscr{Q}_{1} Q_{3}+Q_{2} Q_{4}}{Q_{1}^{2}+Q_{2}^{2}}+2 n \pi\right], \\
j & =1,2, \ldots, k ; n=0,1,2, \cdots .
\end{aligned}
$$

Let

$$
\eta_{0}=\eta_{j 0}^{(0)} \min _{j=1,2, \ldots, k}\left\{\eta_{j}^{0}\right\}, \sigma_{0}=\left.\sigma\right|_{\eta=\eta_{0}}
$$

Next the following assumption is given:

$\left(A_{4}\right) \mathscr{V}_{11} \mathscr{V}_{21}+\mathscr{V}_{12} \mathscr{V}_{22}>0$, where

$$
\left\{\begin{array}{l}
\mathscr{V}_{11}=(2 \rho+2) \sigma_{0}^{2 \rho+1} \cos \frac{(2 \rho+1) \pi}{2}+\epsilon_{1}(2 \rho+1) \sigma_{0}^{2 \rho} \cos \rho \pi+2 \rho \epsilon_{2} \sigma_{0}^{2 \rho-1} \cos \frac{(2 \rho-1) \pi}{2}+2(\rho+2) \sigma_{0}^{\rho+1} \cos \frac{(\rho+1) \pi}{2} \\
+\epsilon_{3}(\rho+1) \sigma_{0}^{\rho} \cos \frac{\rho \pi}{2}+\epsilon_{4} \rho \sigma_{0}^{\rho-1} \cos \frac{(\rho-1) \pi}{2}+\epsilon_{5}+\epsilon_{8} \cos 2 \sigma_{0} \eta_{0}+2 \epsilon_{7} \sin 2 \sigma_{0} \eta_{0} \\
\mathscr{V}_{12}=(2 \rho+2) \sigma_{0}^{2 \rho+1} \sin \frac{(2 \rho+1) \pi}{2}+\epsilon_{1}(2 \rho+1) \sigma_{0}^{2 \rho} \sin \rho \pi+2 \rho \epsilon_{2} \sigma_{0}^{2 \rho-1} \sin \frac{(2 \rho-1) \pi}{2}+2(\rho+2) \sigma_{0}^{\rho+1} \sin \frac{(\rho+1) \pi}{2} \\
+\epsilon_{3}(\rho+1) \sigma_{0}^{\rho} \sin \frac{\rho \pi}{2}+\epsilon_{4} \rho \sigma_{0}^{\rho-1} \sin \frac{(\rho-1) \pi}{2}+2 \sigma_{0}-\epsilon_{8} \sin 2 \sigma_{0} \eta_{0}+2 \epsilon_{7} \cos 2 \sigma_{0} \eta_{0} \\
\mathscr{V}_{21}=2 \sigma_{0}\left(\epsilon_{9}-\epsilon_{7} \sigma_{0}^{2}\right) \cos 2 \sigma_{0} \eta_{0}+2 \sigma_{0}^{2} \epsilon_{8} \sin 2 \sigma_{0} \eta_{0}, \\
\mathscr{V}_{22}=2 \sigma_{0}^{2} \epsilon_{8} \cos 2 \sigma_{0} \eta_{0}-2 \sigma_{0}\left(\epsilon_{9}-\epsilon_{7} \sigma_{0}^{2}\right) \sin 2 \sigma_{0} \eta_{0} .
\end{array}\right.
$$

Lemma 6. Suppose that $s(\eta)=\chi_{1}(\eta)+i \chi_{2}(\eta)$ is the root of equation (16) near $\eta=\eta_{0}$ that satisfies $\chi_{1}\left(\eta_{0}\right)=0$, Proof. By means of equation (16), one derives $\chi_{2}\left(\eta_{0}\right)=\sigma_{0}$; then, one obtains $\operatorname{Re}[d s / d \eta]_{\eta=\eta_{0}, \sigma=\sigma_{0}}>0$.

$$
\begin{aligned}
& {\left[(2 \rho+2) s^{2 \rho+1}+\epsilon_{1}(2 \rho+1) s^{2 \rho}+2 \rho \epsilon_{2} s^{2 \rho-1}+2(\rho+2) s^{\rho+1}+\epsilon_{3}(\rho+1) s^{\rho}+\epsilon_{4} \rho s^{\rho-1}+2 s+\epsilon_{5}\right] \frac{\mathrm{d} s}{\mathrm{~d} \eta}+\left(2 \epsilon_{7} s+\epsilon_{8}\right) e^{-2 s \eta} \frac{\mathrm{d} s}{\mathrm{~d} \eta}} \\
& \quad-2 e^{-2 s \eta}\left(\frac{\mathrm{d} s}{\mathrm{~d} \eta} \eta+s\right)\left(\epsilon_{7} s^{2}+\epsilon_{8} s+\epsilon_{9}\right)=0,
\end{aligned}
$$

which implies where

$$
\left[\frac{\mathrm{d} s}{\mathrm{~d} \eta}\right]^{-1}=\frac{\mathscr{V}_{1}(s)}{\mathscr{V}_{2}(s)}-\frac{\eta}{s}
$$

$$
\left\{\begin{array}{l}
\mathscr{V}_{1}(s)=(2 \rho+2) s^{2 \rho+1}+\epsilon_{1}(2 \rho+1) s^{2 \rho}+2 \rho \epsilon_{2} s^{2 \rho-1}+2(\rho+2) s^{\rho+1}+\epsilon_{3}(\rho+1) s^{\rho}+\epsilon_{4} \rho s^{\rho-1}+2 s+\epsilon_{5}+\left(2 \epsilon_{7} s+\epsilon_{8}\right) e^{-2 s \eta} \\
\mathscr{V}_{2}(s)=2 s e^{-2 s \eta}\left(\epsilon_{7} s^{2}+\epsilon_{8} s+\epsilon_{9}\right) .
\end{array}\right.
$$




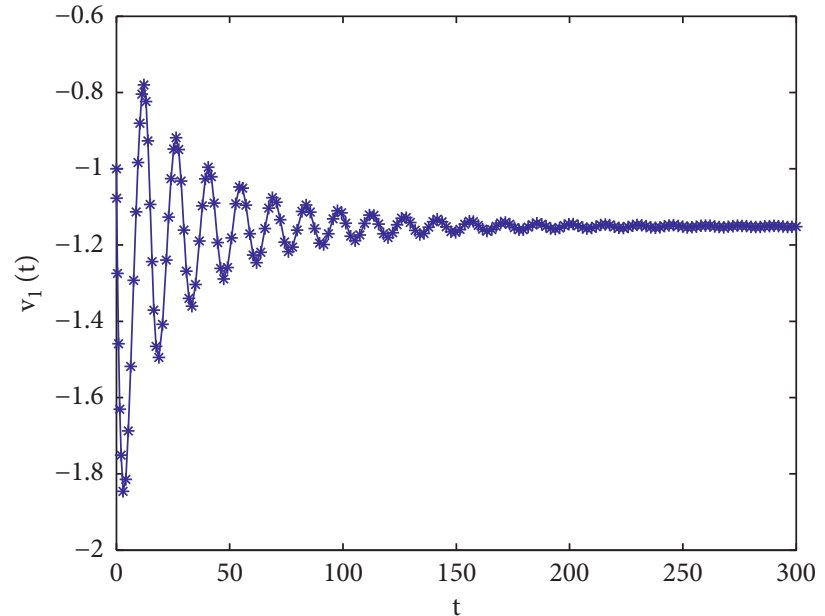

(a)

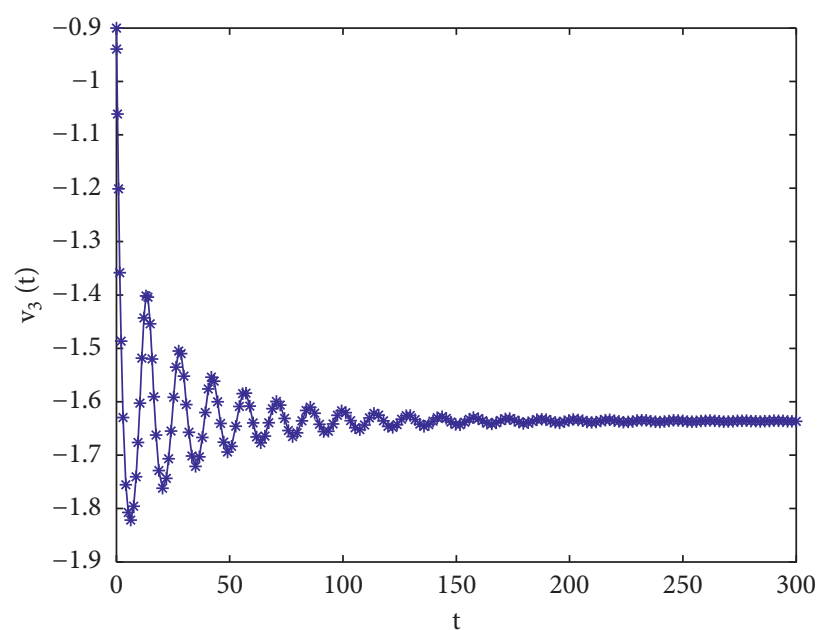

(c)

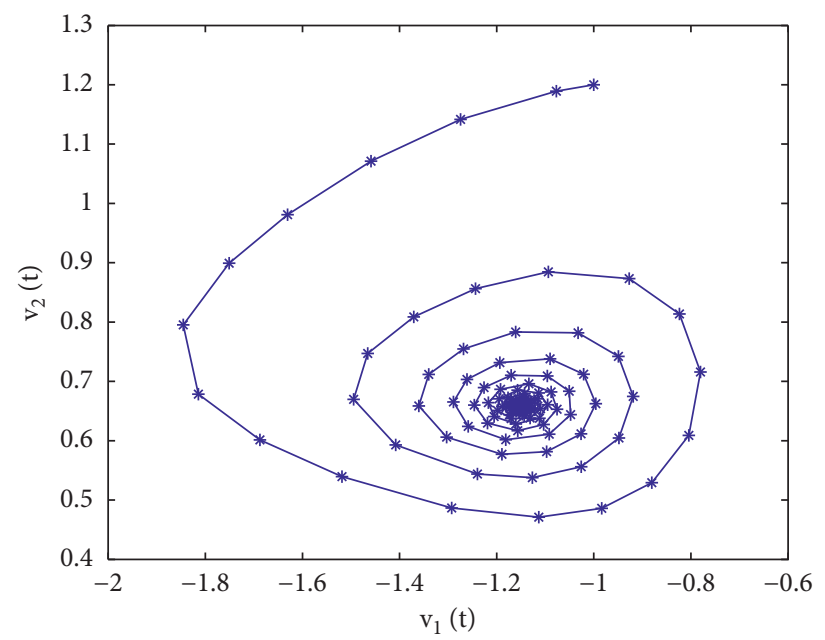

(e)

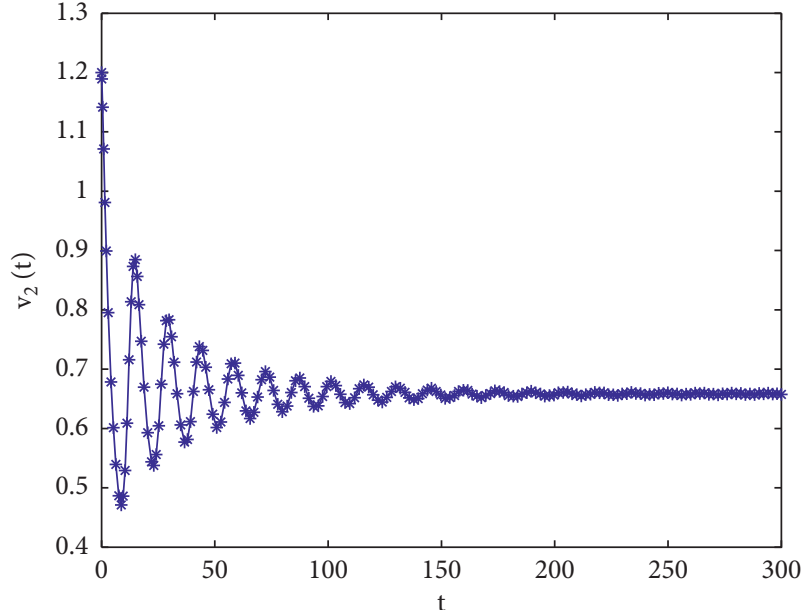

(b)

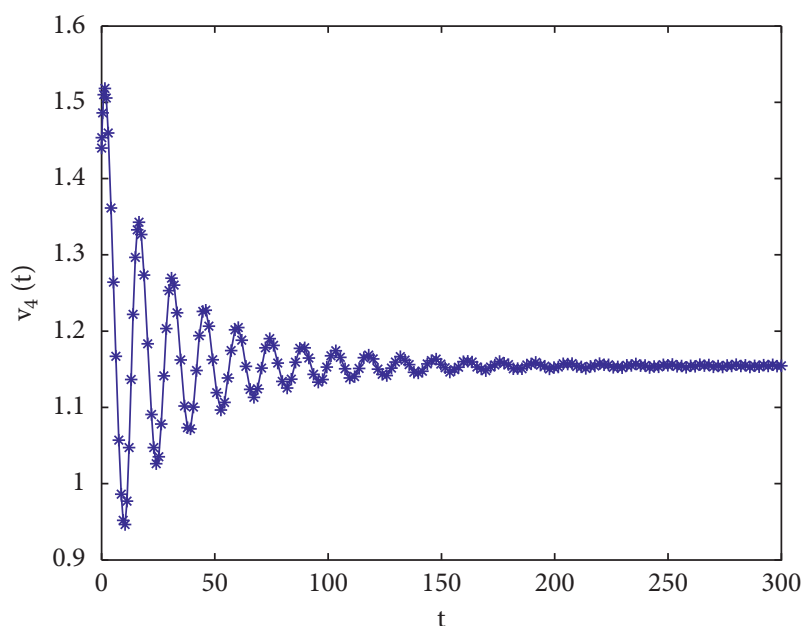

(d)

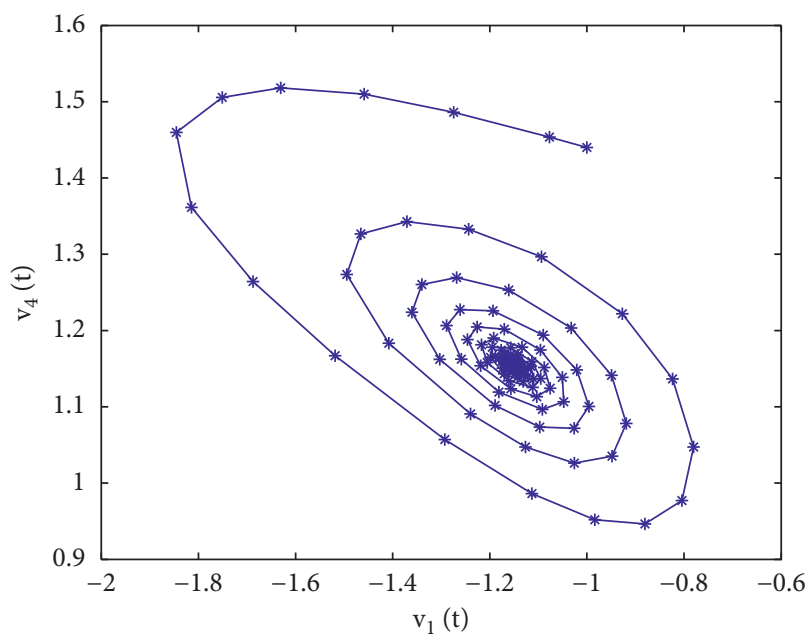

(f)

FIgUre 1: Continued. 


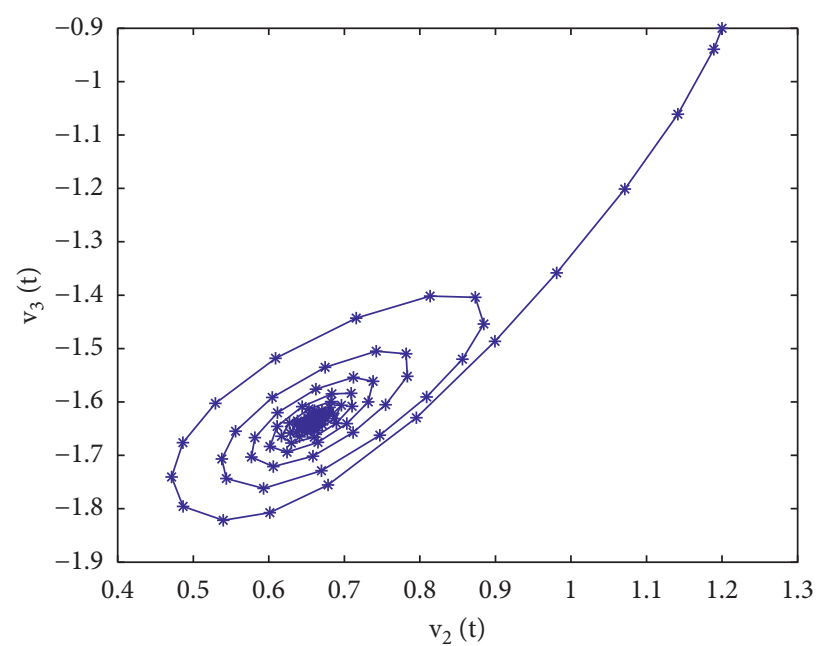

(g)

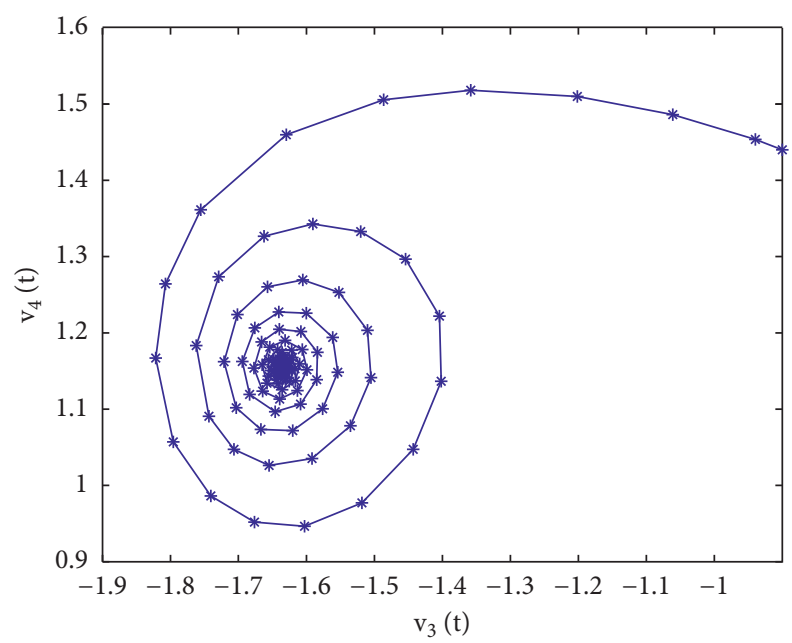

(i)

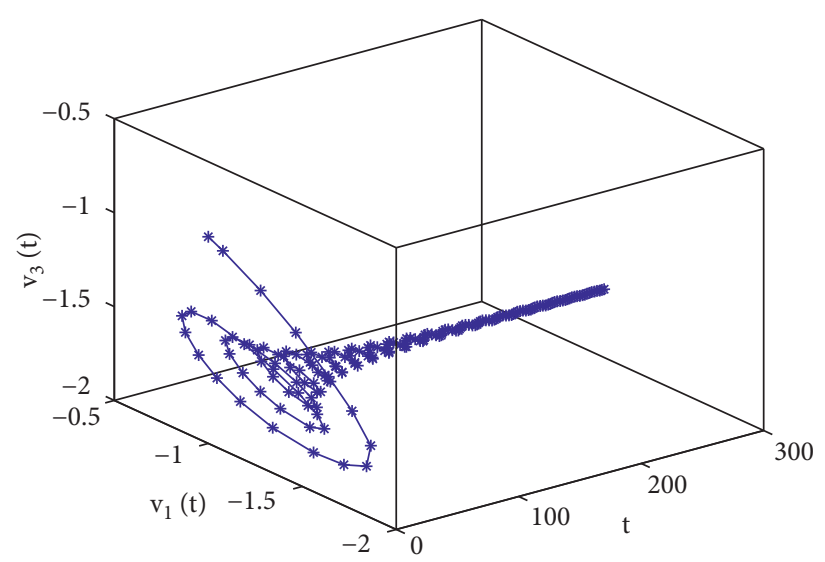

(k)

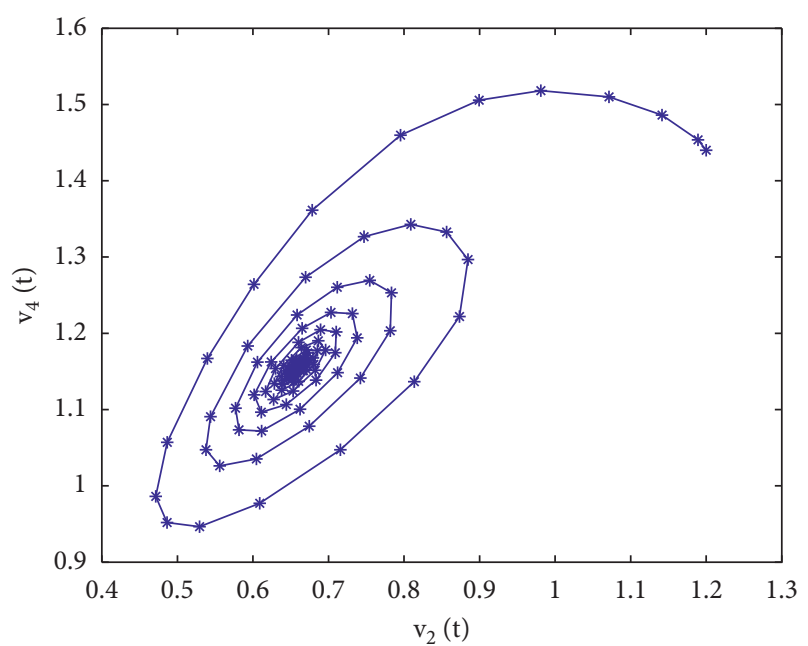

(h)

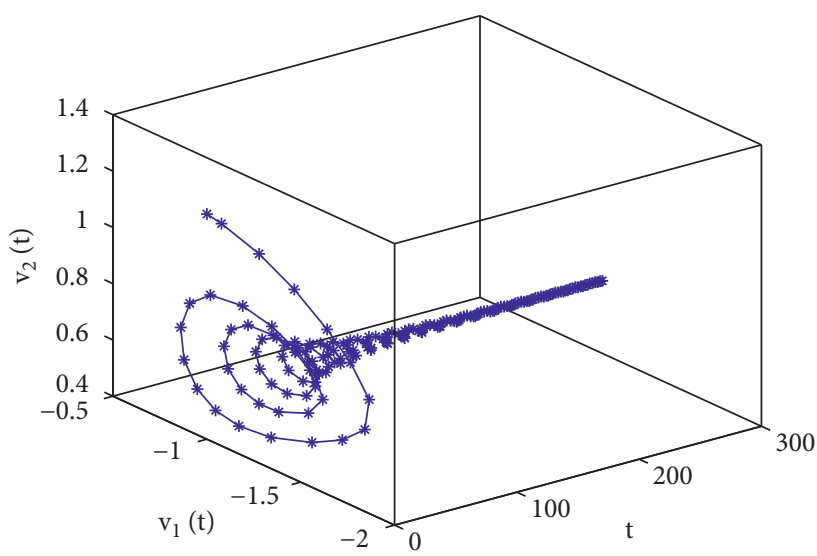

(j)

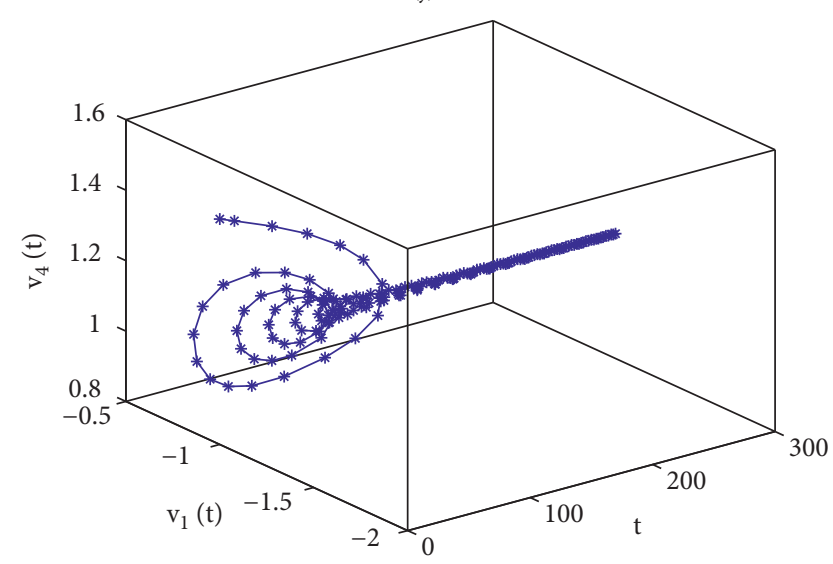

(1)

FIGURE 1: Continued. 


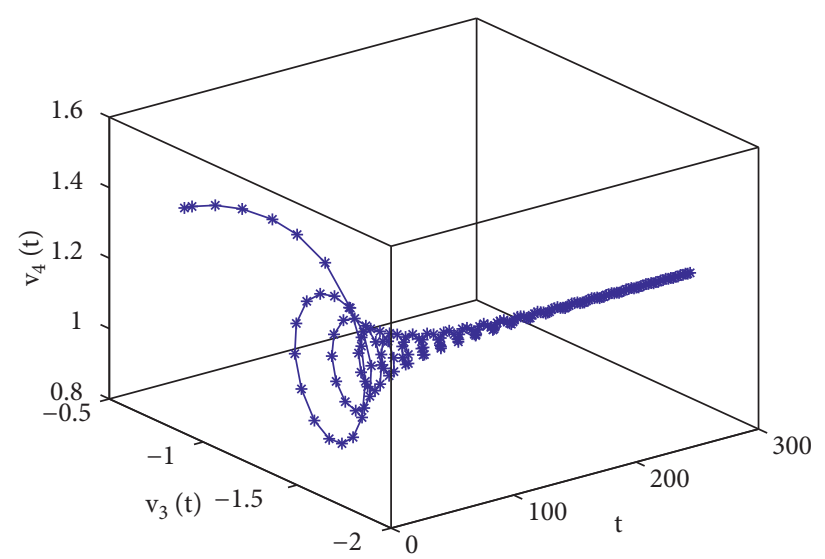

$(\mathrm{m})$

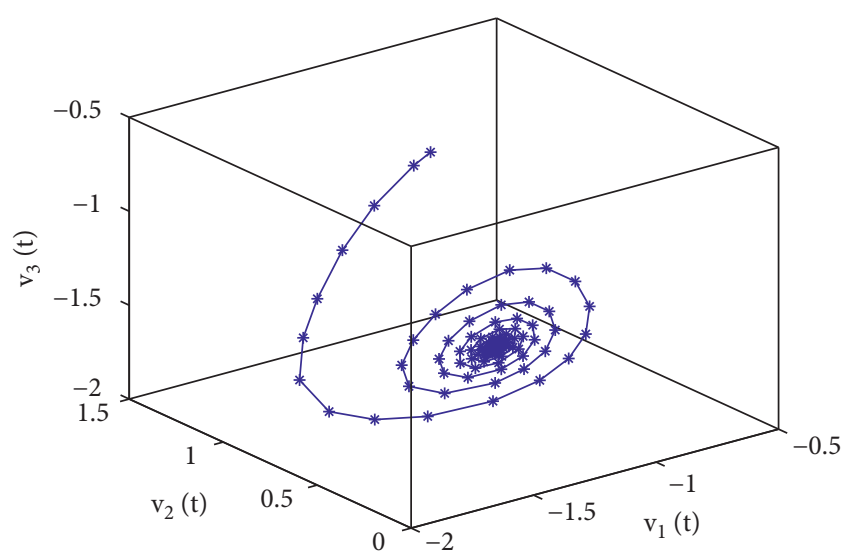

(n)

FIgURE 1: The stable behavior of system (37) when $\eta=0.38<\eta_{0}=0.41$.

Therefore,

$\operatorname{Re}\left[\frac{\mathrm{d} s}{\mathrm{~d} \eta}\right]_{\eta=\eta_{0}, \sigma=\sigma_{0}}^{-1}=\operatorname{Re}\left[\frac{\mathscr{V}_{1}(s)}{\mathscr{V}_{2}(s)}\right]_{\eta=\eta_{0}, \sigma=\sigma_{0}}=\frac{\mathscr{V}_{11} \mathscr{V}_{21}+\mathscr{V}_{12} \mathscr{V}_{22}}{\mathscr{V}_{21}^{2}+\mathscr{V}_{22}^{2}}$.

By virtue of $\left(A_{4}\right)$, we get

$$
\operatorname{Re}\left[\frac{\mathrm{d} s}{\mathrm{~d} \eta}\right]_{\eta=\eta_{0}, \sigma=\sigma_{0}}^{-1}>0 .
$$

The proof finishes.

Based on the exploration above, the following results can be easily established.

Theorem 1. If assumptions $\left(A_{1}\right)-\left(A_{4}\right)$ are satisfied and all the roots $\lambda$ of equation (15) satisfy $|\arg (\lambda)|>(\pi / 2 \xi)$, then the zero equilibrium point of system (2) is locally asymptotically stable when $\eta$ lies in $\left[0, \eta_{0}\right)$ and a Hopf bifurcation arises near the zero equilibrium point when $\eta=\eta_{0}$.

Remark 1. Karaoğlu et al. [29] studied the stability behavior and Hopf bifurcation for integer-order neural network models concerning distributed delays and discrete delays. In this paper, we mainly deal with the stability behavior and Hopf bifurcation for fractional-order neural network models concerning distributed delays and discrete delays. The research method of Karaoğlu et al. [29] cannot be directly utilized to handle the fractional-order one. Based on this idea, we think that the study complements the research of Karaoğlu et al. [29].

Remark 2. Although many authors have studied the Hopf bifurcation issue of delayed fractional-order neural networks, all the equations of delayed fractional-order neural networks are fractional-order cases. In our study, neural network system (2) involves integer-order operator and fractional-order operator. So, there are many differences in research method. Furthermore, so far, there are only very few publications concerning this topic. Based on this idea, we believe that our research enriches the bifurcation theory of fractional-order differential equation.

\section{Simulation Figures}

Consider the system as follows:

$\left\{\begin{array}{l}\frac{\mathrm{d} v_{1}^{\rho}(t)}{\mathrm{d} t^{\rho}}=-v_{1}(t)-0.5 \tanh \left(v_{3}(t)\right)-1.8 \tanh \left(v_{2}\left(t-\eta_{2}\right)\right) \\ \frac{\mathrm{d} v_{2}^{\rho}(t)}{\mathrm{d} t^{\rho}}=-v_{2}(t)+1.5 \tanh \left(v_{1}\left(t-\eta_{1}\right)\right)+1.7 \tanh \left(v_{4}(t)\right), \\ \frac{\mathrm{d} v_{3}(t)}{\mathrm{d} t}=-0.5 v_{3}(t)+0.5 v_{1}(t), \\ \frac{\mathrm{d} v_{4}(t)}{\mathrm{d} t}=-0.5 v_{4}(t)+0.5 v_{2}(t) .\end{array}\right.$

Obviously system (37) possesses a unique zero equilibrium point. Choose $\rho=0.78=39 / 50$ which implies that $\zeta=39, \xi=50$. By virtue of Matlab software, we derive $\sigma_{0}=$ 2.113, $\eta_{0}=0.41, \epsilon_{23}=-0.4317, \quad \mathscr{V}_{11}=0.3368, \mathscr{V}_{12}=$ $0.4251, \mathscr{V}_{21}=-0.3128, \mathscr{V}_{22}=0.5035$. Then, $\mathscr{V}_{11} \mathscr{V}_{21}+\mathscr{V}_{12}$ $\mathscr{V}_{22}=0.1087>0$. In addition, all the roots $(\lambda)$ of equation (15) satisfy $|\arg (\lambda)|>(\pi / 2 \xi)$. Thus, all conditions in Theorem 1 are fulfilled. So, the zero equilibrium point of system (37) is locally asymptotically stable state when $\eta$ lies in $[0,0.41)$. To verify this fact, we choose $\eta=0.38<\eta_{0}=0.41$. The software simulation figures are given in Figure 1. It follows from Figure 1 that four neuron states $v_{1}, v_{2}, v_{3}$, and $v_{4}$ are to be closed to 0 . Figure 1 includes 14 subfigures (Figures 1(a)-1(n)). Different subfigures show the relation of corresponding variables. If $\eta$ crosses $\eta_{0}=0.41$, then the delayed-induced Hopf bifurcation arises near $(0,0,0,0)$. To verify this fact, we choose $\eta=0.5>\eta_{0}=0.41$. The software simulation figures are given in Figure 2. Figure 2 includes 14 subfigures (Figures 2(a)-2(n)). Different subfigures show the relation of corresponding variables. It follows from Figure 2 


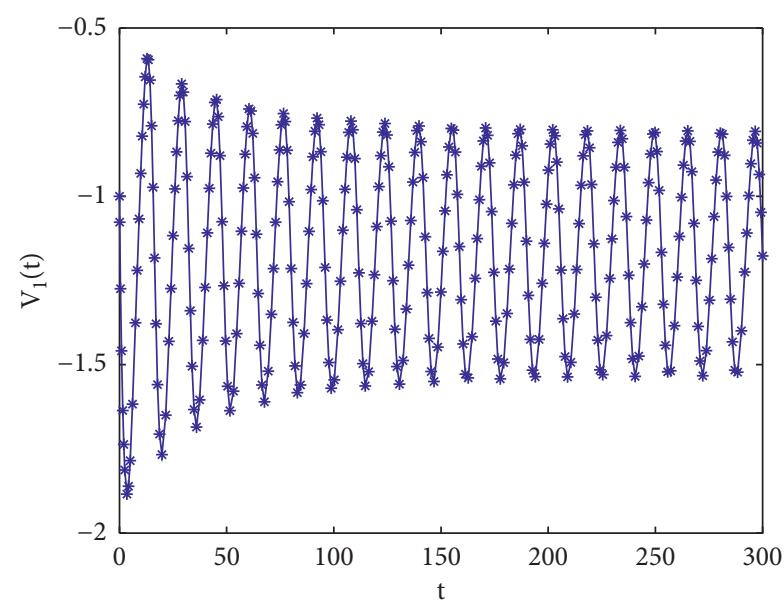

(a)

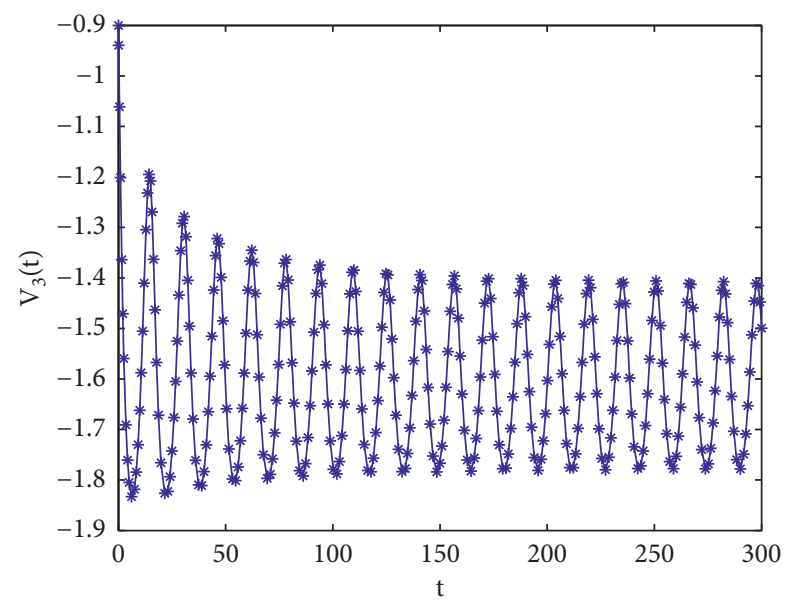

(c)

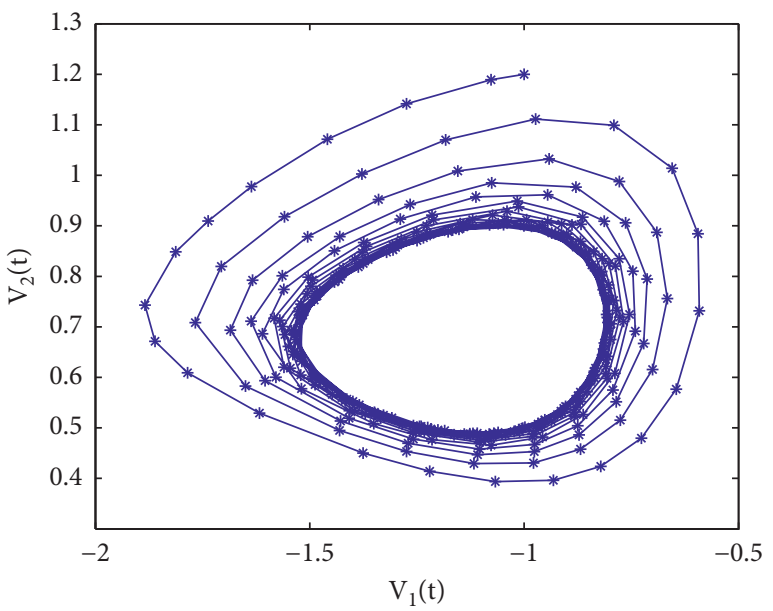

(e)

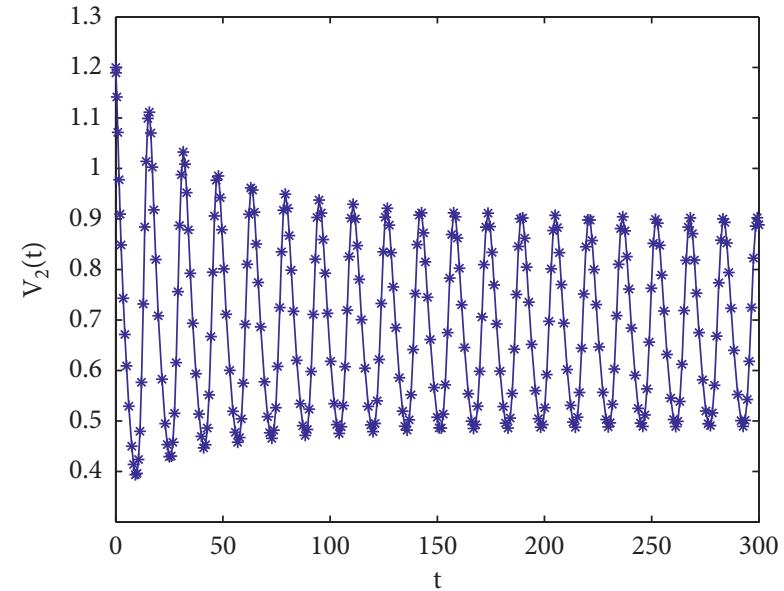

(b)

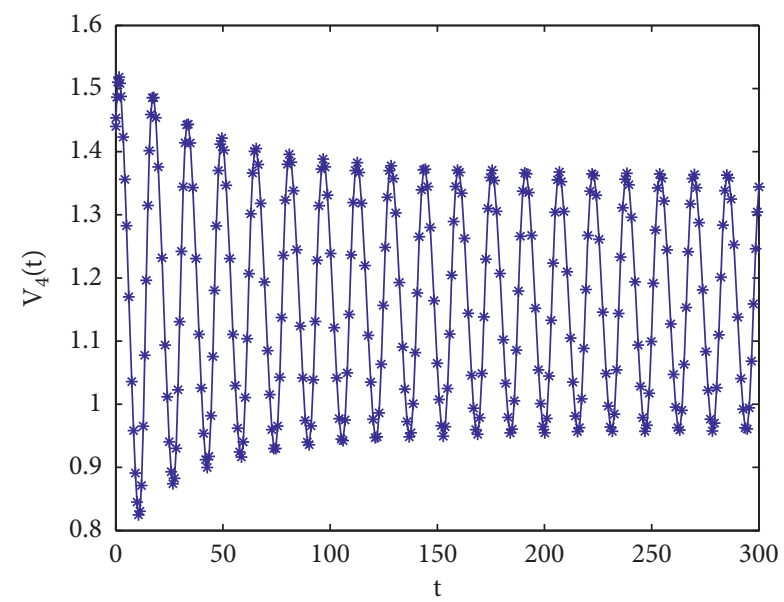

(d)

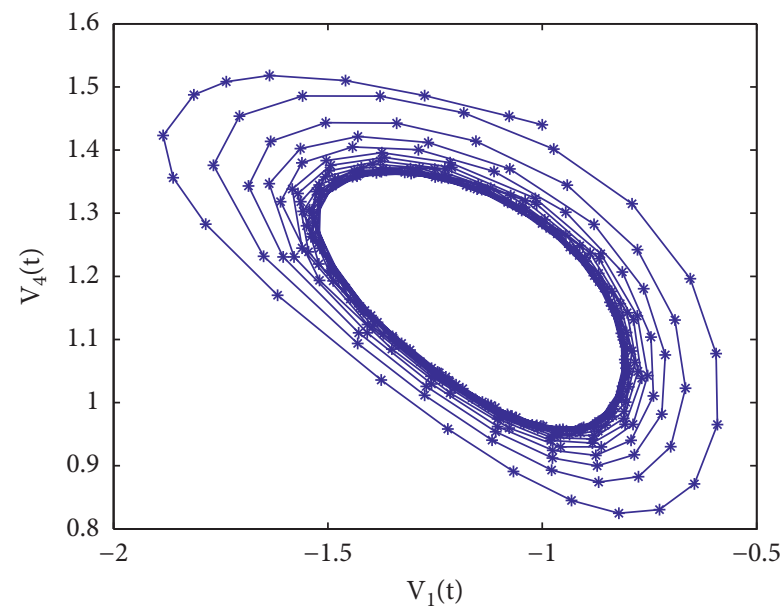

(f)

FIGURE 2: Continued. 


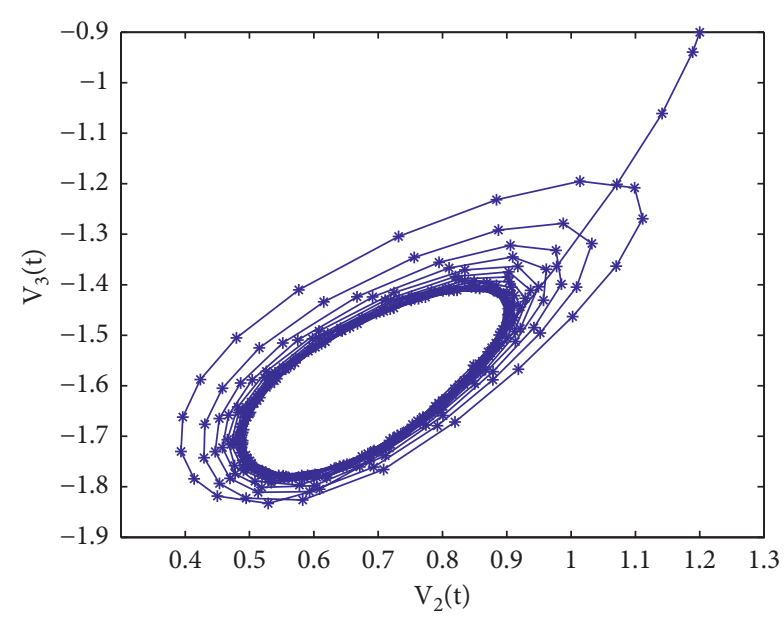

(g)

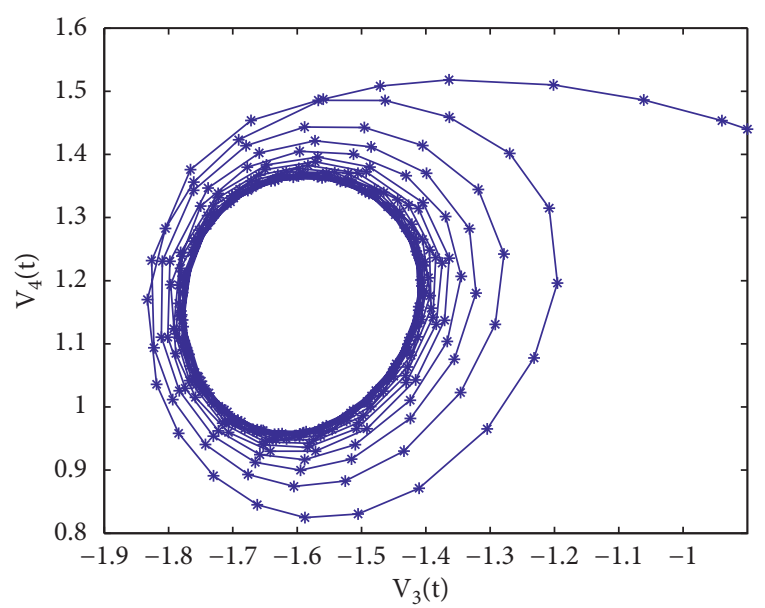

(i)

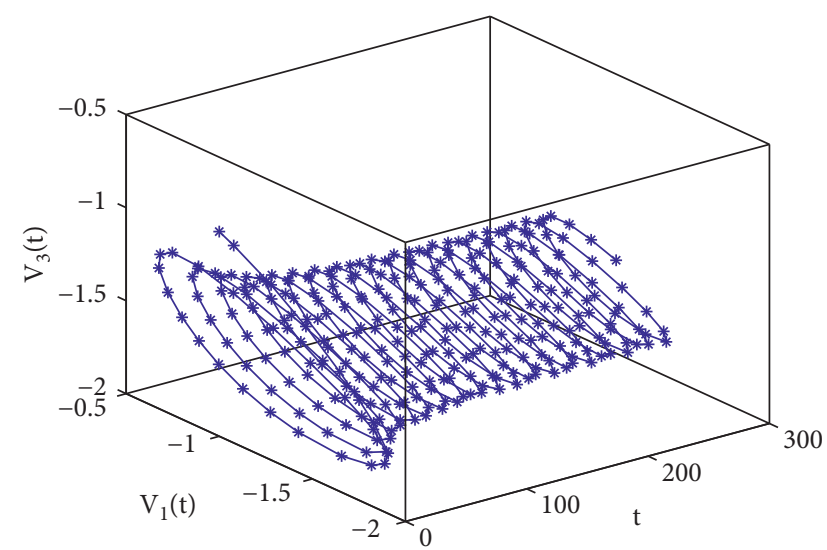

(k)

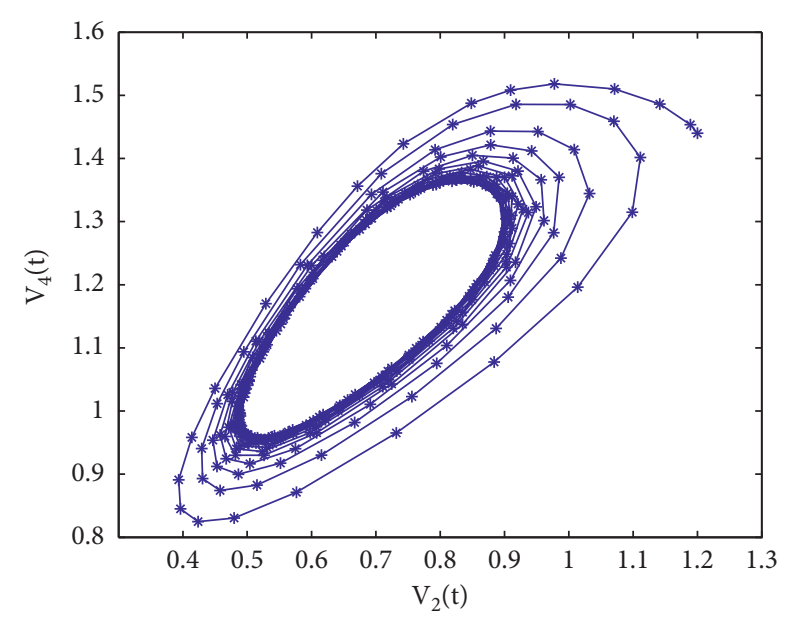

(h)

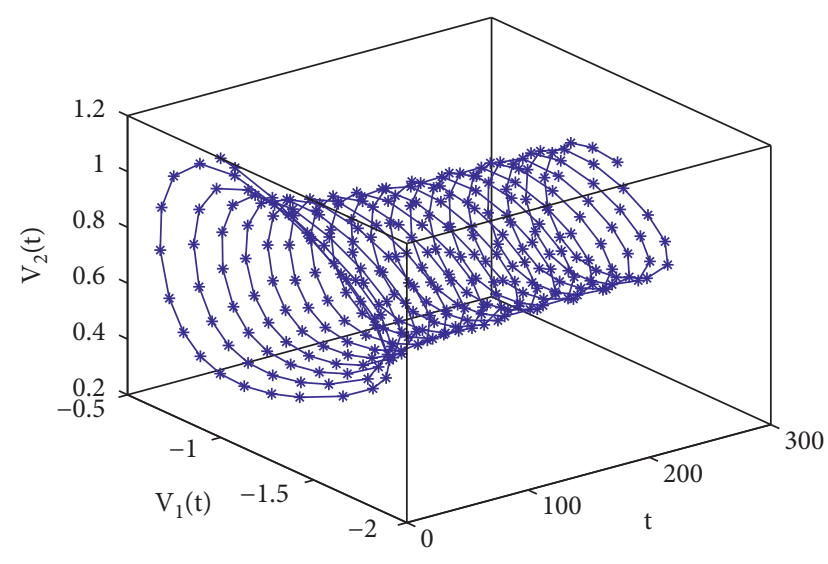

(j)

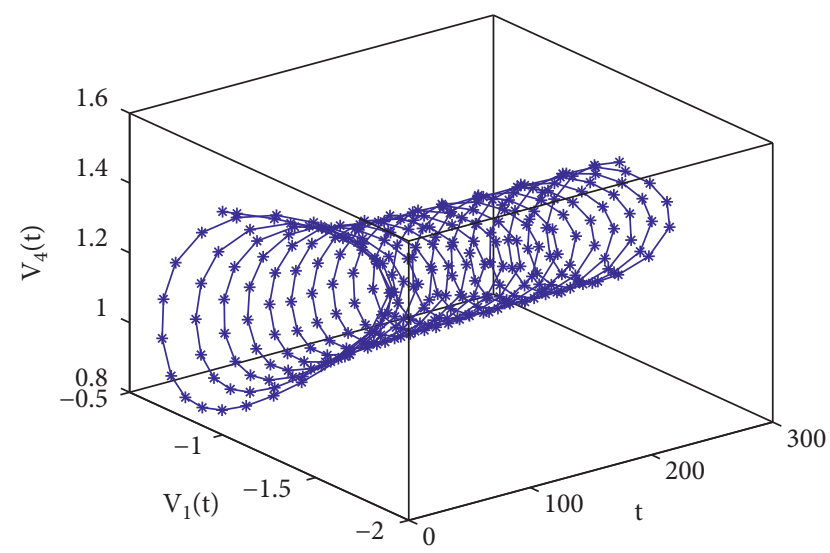

(l)

FIgURE 2: Continued. 


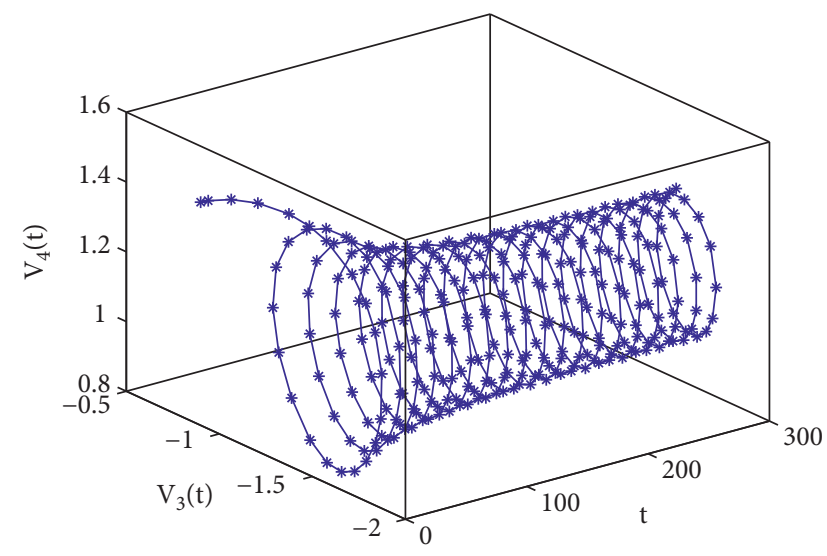

(m)

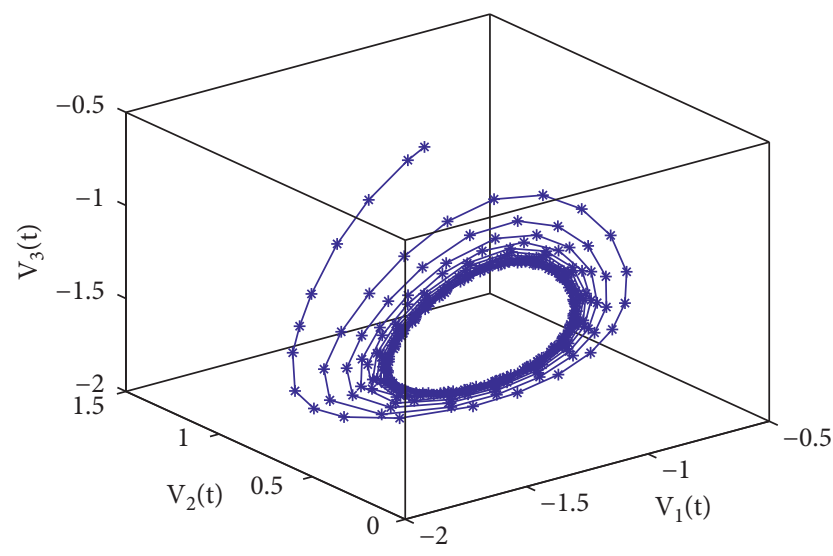

(n)

Figure 2: Hopf bifurcation phenomenon of system (37) when $\eta=0.5>\eta_{0}=0.41$.

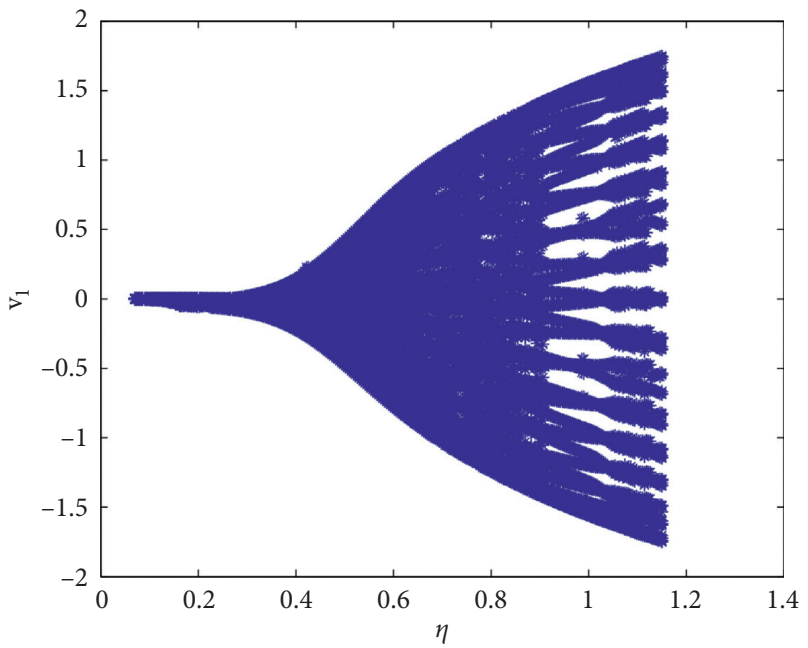

FIGURE 3: Bifurcation graph of system (37): $\eta-v_{1}$.

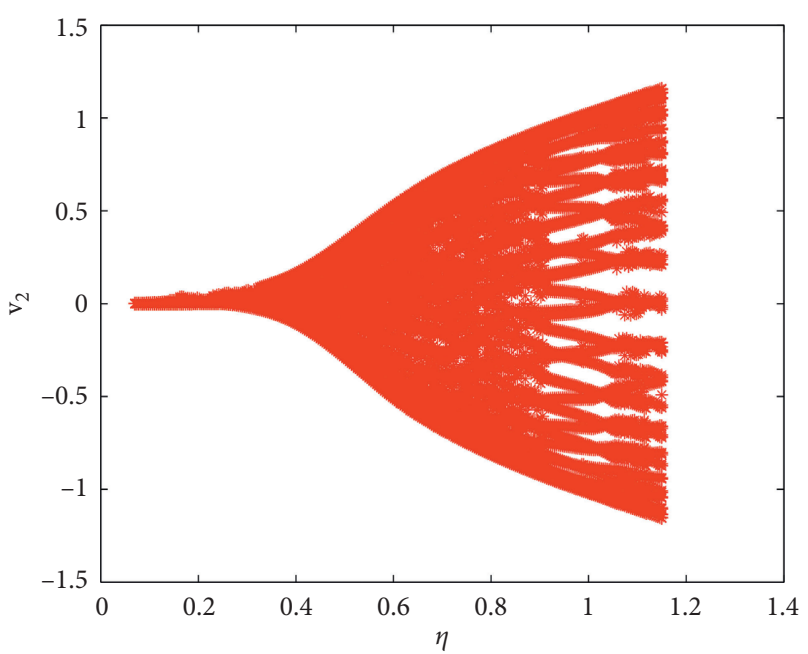

FIgURE 4: Bifurcation graph of system (37): $\eta-v_{2}$. 


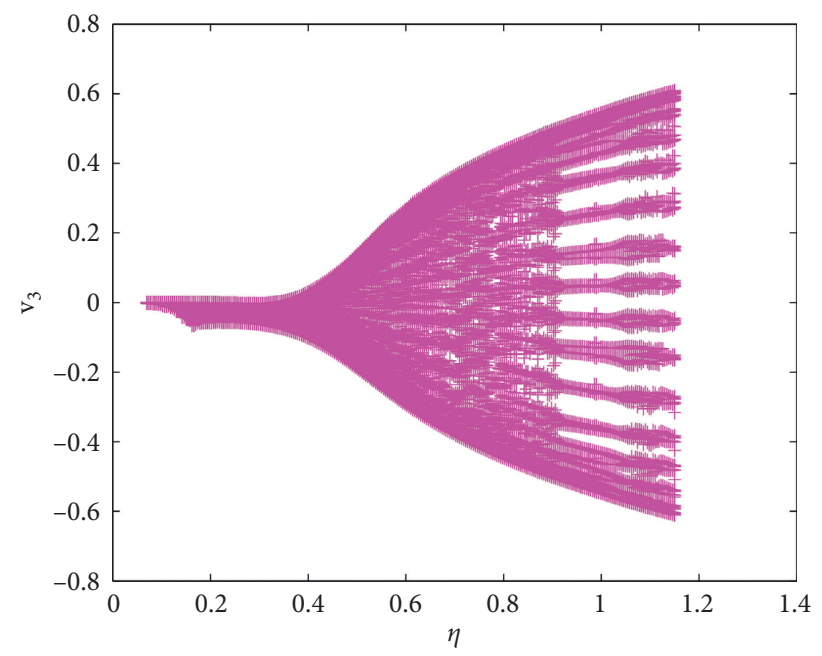

FIGURE 5: Bifurcation graph of system (37): $\eta-v_{3}$.

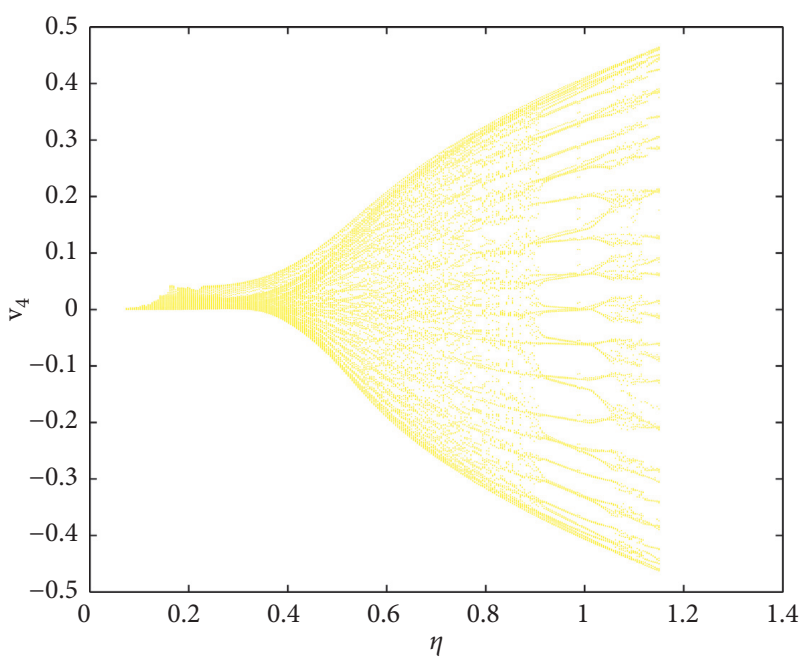

FIGURE 6: Bifurcation graph of system (37): $\eta-v_{4}$.

that four states of four neurons will display the periodic oscillatory behavior. Furthermore, the bifurcation graphs are given in Figures 3-6, which show that the bifurcation value is 0.41 intuitively.

\section{Conclusions}

As is known to us, the stability behavior and Hopf bifurcation nature of many different neural networks are important topics in delayed dynamical systems. In this present study, the stability behavior and the emergence of Hopf bifurcation for fractional-order neural networks involving different types of delays have been investigated in detail. Making use of a suitable variable substitution, novel equivalent fractional-order forms of the involved delayed neural network models are built. Taking advantage of stability theory and bifurcation knowledge of fractional-order differential equation, a novel delay-independent bifurcation criterion for the fractional-order neural networks involving different types of delays is presented. The investigation indicates that time delay plays a significant role in stabilizing and controlling Hopf bifurcation for the involved fractionalorder delayed neural networks. The research method can help us deal with the Hopf bifurcation issue of many other neural networks involving distributed delays in the near future. In this paper, we only consider the effect of the sum of two delays $\eta_{1}$ and $\eta_{2}$ on bifurcation of the involved system. In the coming work, we will deal with the impact of two different delays $\eta_{1}$ and $\eta_{2}$ on bifurcation of the involved system.

\section{Data Availability}

No data were used to support this study.

\section{Conflicts of Interest}

The authors declare that they have no conflicts of interest. 


\section{Acknowledgments}

This study was supported by the Innovation Exploration and Academic New Seedling Project of Guizhou University of Finance and Economics ([2017]5736-025) and Guizhou Province University Science and Technology Top Talents Project (KY[2018]047).

\section{References}

[1] A. Chaouki and A. El Abed, "Finite-time and fixed-time synchronization of inertial neural networks with mixed Delays," Journal of Systems Science and Complexity, vol. 34, no. 1, pp. 206-235, 2021.

[2] C. Xu, M. Liao, P. Li, Z. Liu, and S. Yuan, "New results on pseudo almost periodic solutions of quaternion-valued fuzzy cellular neural networks with delays," Fuzzy Sets and Systems, vol. 411, pp. 25-47, 2021.

[3] C. Aouiti and I. B. Gharbia, "Piecewise pseudo almost-periodic solutions of impulsive fuzzy cellular neural networks with mixed delays," Neural Processing Letters, vol. 51, no. 2, pp. 1201-1225.

[4] Y. Cao, S. Ramajayam, R. Sriraman, and R. Samidurai, "Leakage delay on stabilization of finite-time complex-valued BAM neural network: decomposition approach," Neurocomputing, vol. 463, pp. 505-513, 2021.

[5] Y. Gan, C. Liu, H. Peng, F. Liu, and H. Rao, "Anti-synchronization for periodic BAM neural networks with Markov scheduling protocol," Neurocomputing, vol. 417, no. 5, pp. 585-592, 2020.

[6] Y. Zhao, S. Ren, and J. Kürths, "Synchronization of coupled memristive competitive BAM neural networks with different time scales," Neurocomputing, vol. 427, pp. 110-117, 2021.

[7] F. Lin and Z. Zhang, "Global asymptotic synchronization of a class of BAM neural networks with time delays via integrating inequality techniques," Journal of Systems Science and Complexity, vol. 33, no. 2, pp. 366-382, 2020.

[8] K. Ding, N.-J. Huang, and X. Xu, "Global robust exponential stability of interval BAM neural network with mixed delays under uncertainty," Neural Processing Letters, vol. 25, no. 2, pp. 127-141, 2007.

[9] T. Hou, Y. Liu, and F. Deng, "Stability for discrete-time uncertain systems with infinite Markov jump and time-delay," Science China Information Sciences, vol. 64, no. 5, Article ID 152202, 2021.

[10] L. Zhou, "Global exponential dissipativity of impulsive recurrent neural networks with multi-proportional delays," Neural Processing Letters, vol. 53, no. 2, pp. 1435-1452, 2021.

[11] J. Lin, R. Xu, and L. Li, "Spatio-temporal synchronization of reaction-diffusion BAM neural networks via impulsive pinning control," Neurocomputing, vol. 418, pp. 300-313, 2020.

[12] D. Li, Z. Zhang, and X. Zhang, "Periodic solutions of discretetime Quaternion-valued BAM neural networks," Chaos, Solitons \& Fractals, vol. 138, Article ID 110144, 2020.

[13] M. Yan, J. Jian, and S. Zheng, "Passivity analysis for uncertain BAM inertial neural networks with time-varying delays," Neurocomputing, vol. 435, pp. 114-125, 2021.

[14] M. Syed Ali, G. Narayanan, V. Shekher, A. Alsaedi, and B. Ahmad, "Global Mittag-Leffler stability analysis of impulsive fractional-order complex-valued BAM neural networks with time varying delays," Communications in Nonlinear Science and Numerical Simulation, vol. 83, Article ID 105088, 2020.
[15] E. Arslan, G. Narayanan, M. S. Ali, S. Arik, and S. Saroha, "Controller design for finite-time and fixed-time stabilization of fractional-order memristive complex-valued BAM neural networks with uncertain parameters and time-varying delays," Neural Networks, vol. 130, pp. 60-74, 2020.

[16] C. Xu, M. Liao, P. Li, Y. Guo, Q. Xiao, and S. Yuan, "Influence of multiple time delays on bifurcation of fractional-order neural networks," Applied Mathematics and Computation, vol. 361, pp. 565-582, 2019.

[17] J. Xiao, S. Wen, X. Yang, and S. Zhong, "New approach to global Mittag-Leffler synchronization problem of fractionalorder quaternion-valued BAM neural networks based on a new inequality," Neural Networks, vol. 122, pp. 320-337, 2020.

[18] A. Pratap, R. Raja, J. Cao, F. A. Rihan, and A. R. Seadawy, "Quasi-pinning synchronization and stabilization of fractional order BAM neural networks with delays and discontinuous neuron activations," Chaos, Solitons \& Fractals, vol. 131, Article ID 109491, 2020.

[19] H. Bao, J. H. Park, and J. Cao, "Non-fragile state estimation for fractional-order delayed memristive BAM neural networks," Neural Networks, vol. 119, pp. 190-199, 2019.

[20] C. Xu, Z. Liu, M. Liao, P. Li, Q. Xiao, and S. Yuan, "Fractionalorder bidirectional associate memory (BAM) neural networks with multiple delays: the case of Hopf bifurcation," Mathematics and Computers in Simulation, vol. 182, pp. 471-494, 2021.

[21] S. Djilali, B. Ghanbari, S. Bentout, and A. Mezouaghi, "TuringHopf bifurcation in a diffusive mussel-algae model with timefractional-order derivative," Chaos, Solitons \& Fractals, vol. 138, Article ID 109954, 2020.

[22] W. Hu, D. Ding, Y. Zhang, N. Wang, and D. Liang, "Hopf bifurcation and chaos in a fractional order delayed memristor-based chaotic circuit system," Optik, vol. 130, pp. 189-200, 2017.

[23] C. Xu, M. Liao, P. Li, Y. Guo, and Z. Liu, "Bifurcation properties for fractional order delayed BAM neural networks," Cognitive Computation, vol. 13, no. 2, pp. 322-356, 2021.

[24] A. S. Deshpande, V. Daftardar-Gejji, and Y. V. Sukale, "On Hopf bifurcation in fractional dynamical systems," Chaos, Solitons \& Fractals, vol. 98, pp. 189-198, 2017.

[25] C. Xu, M. Liao, P. Li, and S. Yuan, "Impact of leakage delay on bifurcation in fractional-order complex-valued neural networks," Chaos, Solitons \& Fractals, vol. 142, Article ID 110535, 2021.

[26] Q. Cao, X. Long, and X. Long, "New convergence on inertial neural networks with time-varying delays and continuously distributed delays," AIMS Mathematics, vol. 5, no. 6, pp. 5955-5968, 2020.

[27] B. Zhou and Q. Song, "Stability and Hopf bifurcation analysis of a tri-neuron BAM neural network with distributed delay," Neurocomputing, vol. 82, pp. 69-83, 2012.

[28] I. Ncube, "Stability switching and Hopf bifurcation in a multiple-delayed neural network with distributed delay," Journal of Mathematical Analysis and Applications, vol. 407, no. 1, pp. 141-146, 2013.

[29] E. Karaoğlu, E. Yilmaz, and H. Merdan, "Hopf bifurcation analysis of coupled two-neuron system with discrete and distributed delays," Nonlinear Dynamics, vol. 85, pp. 10391051, 2016.

[30] I. Podlubny, Fractional Differential Equations, Academic Press, New York, NY, USA, 1999.

[31] D. Matignon, "Stability results for fractional differential equations with applications to control processing," 
Computational Engineering in Systems Applications, vol. 2, pp. 963-968, 1996.

[32] X. Wang, Z. Wang, and J. Xia, "Stability and bifurcation control of a delayed fractional-order eco-epidemiological model with incommensurate orders," Journal of the Franklin Institute, vol. 356, no. 15, pp. 8278-8295, 2019.

[33] W. Deng, C. Li, and J. Lü, "Stability analysis of linear fractional differential system with multiple time delays," Nonlinear Dynamics, vol. 48, no. 4, pp. 409-416, 2007. 